\title{
MDP-based Vehicular Network Connectivity Model for VCC Management
}

\author{
Abubakar Saad, BSc
}

Computer Science

\author{
Submitted in partial fulfilment \\ of the requirements for the degree of
}

\begin{abstract}
Master of Science
Faculty of Mathematics and Science, Brock University St. Catharines, Ontario
\end{abstract}




\section{Abstract}

Vehicular Cloud computing is a new paradigm in which vehicles collaboratively exchange data and resources to support services and problem-solving in urban environments. Characteristically, such Clouds undergo severe challenging conditions from the high mobility of vehicles, and by essence, they are rather dynamic and complex. Many works have explored the assembling and management of Vehicular Clouds with designs that heavily focus on mobility. However, a mobility-based strategy relies on vehicles' geographical position, and its feasibility has been questioned in some recent works. Therefore, we present a more relaxed Vehicular Cloud management scheme that relies on connectivity. This work models uncertainty and considers every possible chance a vehicle may be available through accessible communication means, such as vehicle-to-everything (V2X) communications and the vehicle being in the range of road-side units (RSUs) for data transmissions. We propose an markov-decisision process (MDP) model to track vehicles' connection status and estimate their reliability for data transmissions. Also, from analyses, we observed that higher vehicle connectivity presents a trace of repeated connection patterns. We reinforce the connectivity status by validating it through an availability model to distinguish the vehicles which support high availability regardless of their positioning. The availability model thus determines the suitability of the MDP model in a given environment. 


\section{Acknowledgements}

I like to extend my gigantic thanks to Professor Robson for guidance, teaching, and being available most of the time to answer my stupid questions. Thiago, for helping understand the software we use for this research. My parents for their supports. My friends and colleagues for making this journey bearable. Thank you 


\section{Contents}

1 Introduction 1

1.1 Thesis Statement . . . . . . . . . . . . . . . . . . 2

1.2 Motivation . . . . . . . . . . . . . . . . 3

1.3 Objectives . . . . . . . . . . . . . . . . . . 3

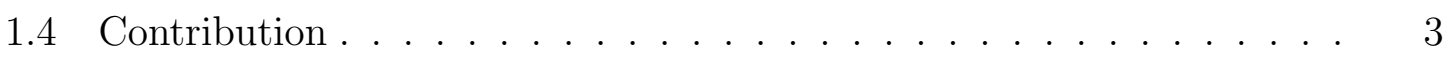

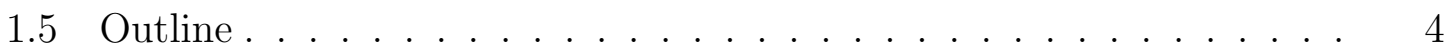

2 Related Works $\quad 5$

2.1 Communication Strategies . . . . . . . . . . . . 6

2.2 Communication Components . . . . . . . . . . . . . . 6

2.3 MANET vs. VANET . . . . . . . . . . . . . . . . 7

2.4 Applications of Vehicular Networks . . . . . . . . . . . 8

2.5 Resource Management . . . . . . . . . . . . . . . 8

2.6 Connection and Communication Stability . . . . . . . . . . 9

2.7 Summary . . . . . . . . . . . . . . . . . . . . . . . . . 13

3 Problem Statement $\quad 15$

4 MDP-based Connectivity Modeling $\quad 18$

4.1 Model . . . . . . . . . . . . . . . . . . . . . . . 18

$4.1 .1 \quad$ State Space . . . . . . . . . . . . . . . . . . . . . . 19

$4.1 .2 \quad$ Action Space . . . . . . . . . . . . . . . . . . . 19

4.1 .3 Transition Probabilities . . . . . . . . . . . . . . 20

4.1 .4 Rewards . . . . . . . . . . . . . . . . . 20

4.1 .5 Policies . . . . . . . . . . . . . . . . 21

4.1.6 Transition Discounts . . . . . . . . . . . . . . . . . 21

4.2 Connectivity Estimation . . . . . . . . . . . . . . . . 21

4.3 VC Management . . . . . . . . . . . . . . . . . . . 23 
5 Connection Cycling \& Pattern Modelling 24

5.1 Availability ... . . . . . . . . . . . . . 24

5.1.1 Definition of Availability . . . . . . . . . . . . . 25

5.1.2 Availability and Connectivity ............. 26

5.1.3 Taxonomy of Availability . . . . . . . . . . . . . 28

5.1 .4 Cycles .............................. 30

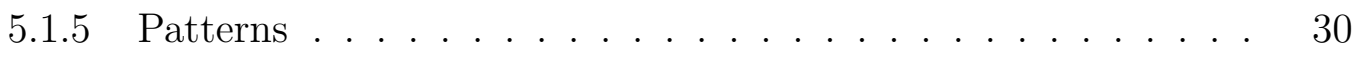

6 Performance Analysis 32

6.1 Scenario . . . . . . . . . . . . . . . . . . . 32

6.2 Traffic Network Topology . . . . . . . . . . . . . . . . . 33

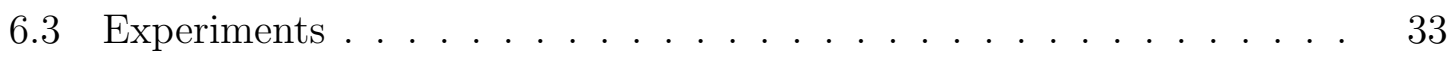

6.4 Performance Analysis of MDP-Model . . . . . . . . . . . . . . 34

6.4.1 Parameters ..................... . . 34

6.4.2 Performance Metrics . . . . . . . . . . . . . . . . . . 35

6.4.3 Results.................... 36

6.5 Performance Analysis of Availability-Model . . . . . . . . . . . . . . 48

6.5.1 Parameters . . . . . . . . . . . . . . 48

6.5.2 Performance Metric . . . . . . . . . . . . . . 50

6.5.3 Results....................... 51

6.6 Summary . . . . . . . . . . . . . . . . . 59

$\begin{array}{lll}7 & \text { Conclusion } & 60\end{array}$

7.1 Summary of Contributions . . . . . . . . . . . . . . . 60

7.2 Future Research Directions . . . . . . . . . . . . . . 61

$\begin{array}{ll}\text { Bibliography } & 67\end{array}$ 


\section{List of Tables}

2.1 Comparison of the Related Works. . . . . . . . . . . . . 13

6.1 Simulation Parameter Settings . . . . . . . . . . . . . . 35

6.2 Simulation Parameter Settings . . . . . . . . . . . . . . 49 


\section{List of Figures}

3.1 Vehicular Clouds in an urban scenario. . . . . . . . . . . . . . 16

5.1 Availability Taxonomy. . . . . . . . . . . . . . . . . . . . . . . 29

5.2 State \& Transition of the Resources. . . . . . . . . . . . . . . 30

6.1 Cologne Metropolitan area used in the simulation analysis. . . . . . 33

6.2 Ranking of vehicle connectivity on a 500-vehicle scenario. . . . . . . 37

6.3 Ranking of vehicle connectivity on a 1000-vehicle scenario. . . . . . 38

6.4 Ranking of vehicle connectivity on a 2000-vehicle scenario. . . . . . 39

6.5 Vehicles head for destination. $(\gamma=1) \ldots \ldots \ldots \ldots$

6.6 Vehicles head for destination. $(\gamma=1) \ldots \ldots \ldots \ldots \ldots$

6.7 MDP-model ranking involving random mobility of vehicles. $(\gamma=1) \cdot 42$

6.8 MDP-model ranking involving random mobility of vehicles. $(\gamma=1) \cdot 42$

6.9 MDP-model ranking involving random mobility of vehicles. $(\gamma=1)$. 43

6.10 Ranking of vehicles in high density vs. lower density area . . . . . . 44

6.11 Node ranking with scenario including LTE Tower $(\gamma=0.1) \ldots \ldots .44$

6.12 Node ranking with scenario including LTE Tower $(\gamma=0.5) . \ldots . \quad \ldots 4$

6.13 Ratio of successful connections. . . . . . . . . . . . . . 46

6.14 Ratio of successful connections show with different position of RSU. . 47

6.15 Ranking from MDP-model. . . . . . . . . . . . . . . . . 52

6.16 Availability Ranking. . . . . . . . . . . . . . . . . . . 53

6.17 Success Ratio. . . . . . . . . . . . . . . . . . . 54

6.18 Success Ratio. . . . . . . . . . . . . . . . . . 55

6.19 Availability Ratio. . . . . . . . . . . . . . . . . 55

6.20 Ranking from MDP-model Worse Case. . . . . . . . . . . . . 56

6.21 Availability Ranking Worse Case. . . . . . . . . . . . . . 57

6.22 Success Ratio. . . . . . . . . . . . . . . . . . . 58

6.23 Availability Ratio. . . . . . . . . . . . . . . . 58 


\section{Chapter 1}

\section{Introduction}

Vehicular Cloud computing (VCC) has been defined as new paradigm where smart and connected vehicles are put together to form a mobile Cloud [10]. Recent technological advancements have driven the attention in creating VCCs. Serving as support, such advancements allowed smart vehicles to contain high processing and storage capacity; these vehicles can also connect and interact among themselves and with the Internet through the use of vehicular networks. By creating a Cloud, these vehicles can collaboratively build a distributed system that extends their own individual capabilities.

The volume of vehicles in urban centers and their highly dynamic mobility position them as valuable resource providers for Smart Cities $[5,10]$. Consequently, in the context of intelligent transportation systems, VCCs can potentially support urban computing in a wide extent, turning itself into a truly attractive but rather complex resource, data, and service management approach to explore. Numerous works have proposed and designed practical services and applications based on Vehicular Clouds [5]. These works include applications for road safety, traffic density, parking lot availability and navigation guidance [37]. Thus, enhancing how such Clouds are assembled and managed is highly rewarding and significantly impacts the community to a great extent.

Coping and dealing with the high mobility of vehicles consist of the great challenge in VCC. Works in Vehicular Cloud Computing and Vehicular Networks rely heavily on precisely modeling the movement and position of vehicles and devices to provide proper resource management. The current management methods for Vehicular Clouds dully depend on mobility, proximity, and position of vehicles [5]. These methods require constant, and sometimes exhaustive, monitoring. This communication management scheme is very costly and prone to errors, impacting the assembling 
of Vehicular Clouds.

Besides high monitoring and control costs, these approaches face situations where there is minimal chance of communication where vehicles stay in range very briefly due to their high speed. Consequently, the short amount of time vehicles are near each other turns the delivery of Cloud services almost impractical. Scientific works [10] have extensively questioned the feasibility of Vehicular Clouds in highly mobile environments, such as urban centers. These works have demonstrated and proven that the contact time of vehicles is much shorter than for providing any services and resources to a requester.

Besides, several approaches have explored connectivity in vehicular networks. Delay Tolerant Networks [9], for instance, have coped with low-density vehicle scenarios to guarantee some level of packet delivery. Many technologies also make use of vertical and horizontal handoffs $[3,8]$ to achieve better networking conditions. These methods explore connectivity opportunities, showing prospects that favorably support vehicles to be reachable.

Therefore, due to the existing drawbacks and challenges of exiting mobility-based approaches, we propose an uncertainty-oriented connectivity model. The proposed model aims at discovering and mapping resources and content independent from the communication endpoints, acting similarly to Content-Centric Networks [27], making use of mobility as a secondary parameter that just enables predictions. The approach provides a more relaxed and flexible method of resource discovery and indexing, increasing the opportunities for forming Clouds and finding content in a rather dynamic distributed environment.

\subsection{Thesis Statement}

Resource management relies on proper modelling of communication/connectivity. In vehicular cloud computing nodes can form networks among themselves or can be connected to the internet via Road Side Unit (RSU). These nodes (vehicles) benefit from being a part of the vehicular networks by either requesting services or providing services to other vehicles. Managing vehicles as resources comes with a price, as vehicles have high mobility, which is the cause the lack of communication stability within the network. In vehicular networks paradigm most models are composed/focused towards mobility based communication, that provides challenge of being mirco-managed. Vehicles demonstrate tendencies of unpredictable movements with mobility model, that renders mobility models difficult to cope with high mobility, when vehicles join or 
leaves the network. Therefore, we purposed connectivity based model, which determines or estimates vehicles that are more suitable for accomplishing task requests based on the 'connectivity status'.

\subsection{Motivation}

Previous works deal with the modeling and estimate of connection stability plainly based on the mobility of nodes/vehicles. This mobility-oriented modelling approach imposes/causes considerable communication and computation overhead for accurateenough estimates. This work thus aims at proposing/exploring a relaxed model based on uncertainty. It focuses on approximating the resources 'connectivity status' within the vehicular network. The connectivity model sought acceptable resources to satisfy the services requests. Regardless of the positioning of these resources within the network, as long as the resources are reachable via orthodox form of communication. This work pushes the boundaries of the connectivity model by not just examining the resources, but authenticating these resources with additional model known as availability model.

\section{$1.3 \quad$ Objectives}

This work aims to define a model that provides accurate connection stability based on 'connectivity status'. This model is expected to scale, not be computation- and communication-intensive, and accurate (close to mobility based). This purposed connectivity based model targets the resources via disparate forms of communication within this dynamic high mobility environment. This work pushes the limits on connectivity model through not just discovering high 'connectivity' resources, but validating these resources through contrasting availability model, which furnishes these resources desirability.

\subsection{Contribution}

An MDP-based model that assesses resources to rank them based on the 'connectivity status'. The vehicular networks with rising density of vehicles is a by-product of higher connectivity among the nodes. The measure of the connectivity status of nodes is validated through the MDP-model. The "preliminary" results of this MDP-model has been published in DS-RT 2020 conference. 
An availability model that measures suitability of the proposed MPD-based model. Availability model illustrates the availability of the resources approximated by MDPmodel. This permits for observation if the availability analysis confirms the suitability of the MDP-model or non suitability of the MDP-model established through the movements of the resources.

\subsection{Outline}

This thesis is organized as follows. Section 2 provides an overview on works about configuration and management of VCCs. Section 3 describes the problem tackled in this work. Section 4 presents our approach to enhance Vehicular Cloud management based on connectivity uncertainty. Section 5 introduces availability model for verfication of "connectivity status" of resources in Vehicular Cloud management. Section 6 describes the experimental evaluations and discusses obtained results. Finally, Section 7 presents the conclusion and future work directions. 


\section{Chapter 2}

\section{Related Works}

There has been revolutionary changes in the automotive industry in last decades, the automobile installed with sophisticated technology provides the drivers access to more features. The vehicles in this day and age considered to be equitable computeron-wheels with the powerful on-board unit, storage capability, smart sensors, gps device and collision radars [5]. Similarly, there have been many advancements in the cloud computing, that have allowed mobile devices to build dynamic Clouds networks. Cloud provides more functionality on resource, which is on-demand, one of the largest providers of cloud computer is Amazon Elastic Computer Cloud (Amazon EC2), also provides dynamic computing capability, scaling the infrastructure, resources on-demand, virtualization technology, Quality of Services and that helps companies to be more focused towards providing applications to the customers rather than building or managing their data centers [5]. Combine the cloud and vehicles to produces vehicular Cloudlet, that is based on the vehicles themselves and these vehicles could also share their data with one another through various means: vehicle-tovehicle (V2V), vehicle-to-infrastructure (V2I), and vehicle-to-everything (V2X) [5]. This allows for computing task on the vehicular Cloudlet if needed be, the stable environment is more of parked vehicle and more dynamic environment includes vehicles in the motion and mobility is high, such as, highways. Those networks are called Vehicle Ad-Hoc Networks (VANETs), these type of networks are formed on the fly. There are other types of networks that evolved from the VANETS, called vehicular cloud computing (VCC), these are designed to complete the day to day transportation tasks, such as, low cost for computing for authorized users, reducing traffic congestions and improve the road safety [5]. The reason VCC are gaining more attractions, it is because they have very similar characteristics to mobile-cloud computing (MCC), as VCC dynamically formed by the vehicles that are close by to 
achieve the task or provided a service and because VCC also differs from the mobileadhoc cloud (MAC), as they work in more high volatile envoriment due to the nature of vehicles moving around or in high mobility [5].

The communication between the nodes happens in three ways: vehicle-to-vehicle $(\mathrm{V} 2 \mathrm{~V})$, vehicle-to-infrastructure (V2I), and vehicle-to-everything (V2X) by using the Wireless Access in the Vehicular Environment (WAVE) [5].

\subsection{Communication Strategies}

$\mathrm{V} 2 \mathrm{~V}$ is based on communication between the vehicles in ad-hoc environment, the communication happens through the multi-hop and within the transmission range of the node and it relays information such as, road status, driving conditions and traffic accidents [5] through the nodes within the network.

V2I communication happens between the vehicles and infrastructure. This type of communication happens to other networks, such as, internet or some sort of cloud services, the bandwidth they require is higher compared to the $\mathrm{V} 2 \mathrm{~V}$ link, because it goes through the RSUs or other gateways and it provides better security for the communication [5]

V2X type of communication takes places between vehicle to anything that is capable of connection, for example, cell phones, IoT devices, etc.... This form of communication is very general, the other forms are subset of this type of communication, such as, V2I, V2V, Vehicle-to-pedestrian (V2P), Vehicle-to-device (V2D), and Vehicle-to-grid (V2G) [5].

\subsection{Communication Components}

The Dedicated Short-Range Communication (DSRC) protocol lies on the 5.9 Ghz frequency band, this allow V2V communication [1]. The major components of the communication consist of onboard-unit (OBU), roadside unit (RSU), and certification authorities (CA) in the vehicular network [5]. On the high level, IEEE 802.11p supports both DSRC and Wireless Access in Vehicular Environments (WAVE), which significantly differ from the traditional Wi-Fi and celluar networks [16]. The list of communication media expands as we include LTE and 5G. By leveraging the 5G infrastructure, vehicles can reliably communicate in ultra-high-speed and reduce delays within the network [15]. 
Onboard Unit: The OBU is available in all the vehicles. OBU job is to collect the information from the different sensors within the vehicles, such as, position, speed, acceleration/deceleration and sends this to either another vehicle or RSU, but not only that, its also able to receives, process or store data, and verify the task to avoid the security breaches [5].

Roadsite Unit: The RSU is crucial component in vehicular networks as it allows the communication with the infrastructure and this unit is fixed at a location, usually closer to the intersection. This unit is responsible for collecting and disseminating data related to the traffic conditions, accidents, traffic jams or even the parking spots [5]. Moreover, in order to execute this operations RSU needs at least one networking interface that would allow it to connect to the Internet and also connects it to shortrange wireless communication [5]. Finally, RSU can also acts the gateway for vehicles that require information or services from the Internet or other networks and can also servers as monitoring endpoint [5].

Certification Authority: CA manages security risk in the vehicular networks [20]. This components monitoring the nearby vehicles and asses if the information is from validated authority, such as, municipal transportation department [5]

\subsection{MANET vs. VANET}

VANET are subclass of MANET, they have very different protocols and characteristics in terms of communication, VANETs aim towards more vehicular network [5]. If the projection of the MANET on to VANET would be considered as such, RSUs represents the static nodes and OBUs will represent the mobile node in the ad-hoc environment and the communication will happen through to the 802.11 p protocols [5]. There are some major differences between the two of them:

Pack Transmission: Packets are limited to the area of the coverage distance, which promotes for different type of routing protocols, such as, on-demand routing, tabledriven routing, and hybrid routing to transfer or share the data among the neighbouring nodes or within the network in ad-hoc domain [5]. These MANETs protocols do not work in the VANETs, because it is hard to cope with the high mobility in the ad-hoc vehicular network [5].

Composing Nodes: Vehicular network has much larger volume of nodes that composed as VANETs compare to MANETs, because usually Vehicular network are broken down into smaller regions focused towards the information of local area, such as, services and applications, but neighbouring network could also provide the informa- 
tion if needed as RSU's serve as a gateway [5].

Topology of Vehicular Networks: The topology of vehicular network is based on the road layout and traffic conditions, vehicles have very limited routes to travel in and the predictability of the nodes increases in the vehicular network [5].

Power limitation: MANETs nodes have limited power to compute tasks and its possible to creating the bottlenecks in the system to fetch for other alternatives communication solution, contrary to the VANET's, there is no limitation on OBU to compute the task, OBU can computer complex task and can also store data [5]

\subsection{Applications of Vehicular Networks}

There exist various applications in vehicular networks as the interest of exploring such networks grows. The application in vehicular network can be break down into three categories [5]:

- Application for road safety: The focuses of these type of applications are towards the road safety, which is enabled by the vehicular networking. For example, hazard notifications, collision avoidance and warnings, that can enable users to access information about the weather conditions and traffic accidents [5].

- Applications for traffic efficiency: The aims for these type of applications is to provide support for the drivers and transportation management system [5]. Support to provide with better interactive functionalities in the vehicle, such as, decision-making during driving, collision avoiding assistant, warning for traffic jams, overtaking other vehicles and additional information for decision process [5].

- Applications for passenger comfort: These application targets the passenger or the driver, to provide comfort by leveraging the onboard services, such as, Internet access, messaging, and infotainment [5].

\subsection{Resource Management}

When involving Intelligent Transportation, approaches have tackled resource management to a great extent by exploring challenges originated from the high mobility of vehicles [10]. In the majority of cases and works, we can observe that the core aspects of such approaches consists of dealing with the unpredictability and highly-dynamic 
communication topology changes. As exception to such cases, there are approaches that do not emphasize on mobility - the ones admittedly based on static parked vehicles, such as the service and resource provision of a static VCC in a Fog computing model [28]. Static VCC, when configured with Vehicular Fog Computer (VFC) infrastructure provides the support to nearby vehicular networks for routing and safety message broadcast [28]. The perks for this type of infrastructure are not necessary to deploy new infrastructure, because of the parked vehicles acted as the RSU and support the vehicular network. However, the most common scenarios are dynamic, and works have already proven the non-feasibility of assembling Vehicular Clouds and cooperation while vehicles are moving on the traffic network [10]; the fast pace changing scenario makes inviable for vehicles to sustain plausible connections long enough to fulfill minimum sharing requirements. Even enabling a platoon-oriented Vehicular Micro-Clouds that compose a distributed larger-scale Cloud cannot guarantee long-standing proximity and contact among vehicles. Besides, these approaches rely on overwhelming control to assess the movement of nodes continually.

\subsection{Connection and Communication Stability}

The vehicles network and the connected vehicles have played a important role in the applications of smart transportation systems. This heterogeneous environment consist of vehicles, smart phones, roadside units, etc. This requires the needs for better content delivery protocols and architectures [7]. The content created for these vehicles should be limited to the certain area, because of the nature of the content. The content most likely will be composed of road conditions, traffic congestions or accidents [7]. Hence, this content will only be delivered in the limited area and most vehicle have rigorous QoS requirements for there content [7]. In the beginning the research for data dissemination started of with the static node in the traffic, then carried on the RSU for more range or coverage of the area, then moved on to the dynamic vehicles moving in the different direction and using the direction as the key to disseminate data [7].

The information-centric Network (ICN) presents a very different way that content could be delivered to the network or retrieved from the network [7]. Basically, ICN over comes the shortfalls of traditional networking models by decoupling the physical address of the network structure to the information classification indexing [24]. ICN focused more towards the content that is requested by the users rather than where the content is located, which helps build more of content-based commu- 
nication model rather than the host-based [38]. The content-based networks allow the users to retrieve information from the nearest provider as long as the content is cached or resided at a node, this type of network helps avoid the long trip to the source of the content and fast delivery to the requester [38]. The proposed structure in [38], known as EcoMD, which focused towards the delivery of the video content through the following means: media delivery system (MDS), road side units (RSU) and vehicular nodes (VN). As the means of communication differ between the heterogeneous services, such as, Road Safety to information sharing and infotainment, the requirement for the efficient network structure grows as well for various services can be supportive [24].

Intelligent Transport System (ITS) are formed by the advancement in the mobile networks and resource in vehicles such as, on-board units that are capable of handling such platform. While, Vehicular Ad Hoc Networks are becoming more popular in wireless networks, where vehicles communication happens in multiple ways, such as, vehicle-to-vehicle (V2V), vehicle-to-infrastructure (V2I), and vehicle-to-everything (V2X) [34]. Vehicular Cloud offers same type of services as mobile users receives, such as, Network as a Service (NaaS), Storage as a Services (SaaS), Cooperation as a Service (COaaS), INformation as a Service (INaaS) and Entertainment as a Service (ENaaS) [34]. These services can be offered only because the vehicles have enough computing, storage and processing power to able provides such to the consumer. There are two different types of networks that VC offers, static and dynamic. Static is where the vehicles are parked or in static mode and dynamic is when the vehicles are motion. VCs can be formed in either type, the latter type is difficult to work with as vehicles are constantly on the move. [34] focused towards the dynamic VC's. Dynamic VC's comes with many challenges such as, high mobility of the vehicles, network topology could change any minute, packet transmission might fails as the vehicle might move out of the range of another one vehicle or even the RSU, these challenges increases the difficulty of creating dynamic VC's comparing to static VC's [34]. Finding the content providers in dynamic environment is a difficult task, which means, consumer vehicles (CV) needs to first find stabilized connection to the content providers before even requesting any services and CV should also find what services would be sufficient from the provider [34]. The paper [34] provides the Fuzzy Quantified Service Selection scheme (FQSS), which depends on the stabilized connection, services offers by providers and consumer requests. Moreover, the paper also rank based on the two operators: least satisfactory proportion (lsp) and greatest satisfactory proportion (gsp) [34]. 
The design of methods to properly form and sustain Vehicular Clouds involves a range of several areas, including the underlying networking protocols. Several works have already explored novel Content-centric Networks (CCN) to cope with the dynamic vehicular environments [7], making use of information-oriented paradigm to target the discovery of data more precisely. Similarly, CCN-based service-oriented architectures [24] stand on and may greatly benefit from Vehicular Cloud-based infrastructure to support efficient content-oriented sharing methods. However, the support of CCN requires frequent updates and mobility prediction models to handle communication and connection inconsistencies. Predicting the position of vehicles, a extensively explored approach in vehicular environments, supported the definition of stability of the communication link between vehicles [4]. Equivalently, the link stability metric has been defined in other works [34] according a quantification of wireless link stability based on the movement of vehicles in a rectilinear form. The relative distance, or speed, among vehicles characterizes stability. This particular work extends its definition by adding multi-hop estimation on stability, tackling the $\mathrm{V} 2 \mathrm{~V}$ communication scenarios, which is employed in a fuzzy-based selection system to match with QoS service requests. In summary, mobility models support estimates to benefit the communication VANETs, even enabling approaches focused on the content instead of vehicles to better balance information supply-demand [38].

Vehicular Delay tolerant networks (VDTNs) are new type of vehicular networks that provides the communication to happen between source and the destination, where there is no end-to-end path available, similar to the most vehicular ad-hoc network, where the nodes are mostly mobile and cooperation of each nodes maintains the whole network [9]. The nodes have simple operations, store-carry-and-forward, this breaks down the nodes to be in three different types: mobile, terminal, and relay nodes [9]. Mobile nodes, such as, vehicles, will mostly be in mobility and possibly communicate with the other types of nodes, terminal nodes are static and placed on the edge of the network, their job is to process more heavy task and communicate with other types of network. Lastly, relay nodes are placed at the intersections to create more communication within the network and interact with the mobile nodes [9]. The interaction between the nodes are broken down into two category: control plane and data plane [9]. In the control plane, nodes shares meta information among themselves, such as, speed, buffer speed, destination node), in the data plane, data is formed into the datagram and complied into the bundles, also bundles are moved to the single or multiple destination [9].

Undoubtedly, Vehicular Networks are prone to connection losses. Formal modeling 
and analysis of delay allow the handling of intermittent connectivity in Vehicular Networks in sparse scenarios [36]. Low RSU coverage implicates in connection losses, which are also influenced by the speed and density of vehicles in road segments. The cost in implementing an infrastructure to support V2I communication has motivated works to search for optimal placement of RSUs on a traffic network so that delaysensitive applications are not compromised [19]. Effective handling of connection loss facilitates the implementation of many Cloud applications. With the proliferation of computing-capable devices and vehicles, mobile Crowdsourcing has been explored to enable pervasive Cloud services through outsourcing tasks [29]. The massive number of devices allows for offloading and environment sensing, being more adequate and economical.

There is a firm assumption that Vehicular Clouds will inevitably benefit cooperation and sharing through significantly adaptable Cloud management. Recent works have promoted such Clouds by tackling diverse aspects, such as mobility, heterogeneity, and scheduling. Cluster-based Vehicular Cloud creation and coordination in highly dynamic urban scenarios aim at following platooning-inspired strategies $[22,21]$. Based on this approach, the MDP-based modeling of vehicle mobility attempted to define enhanced resource allocation in Vehicular Clouds [23]. In the same perspective, a mobility model based on Artificial Neural Networks allowed to reduce the impact of sudden movement changes for more efficient resource allocation in Vehicular Clouds [25]. Cluster-based approaches support multi-edge computing [13] where vehicles take the role of service providers. Thus, vehicular gateways relay data, flowing it through the network and being fundamental role players in spreading data; this approach then heavily rely on mobility to select gateways accurately, directly impacting the efficiency in accessing and dissipating data.

Formal models based on mobility traces undertake a more precise selection of Vehicular Cloud hosts to received offloaded tasks, given their required completion times [6, 39]. The diversity in urban environments has motivated the SMDP-based modeling to represent the heterogeneous spectrum of computing capabilities of vehicles and RSU to better assess the availability and offloading strategies [17]. Eventually, Cloud management culminates in resource allocation, so efficient task scheduling is capable of leveraging Fog Computing and providing additional resources to build up distributed Data Centers [33]. Similar to Mobile Cloud computing, heuristic-based placement and scheduling algorithms have been defined to offload tasks and modules from Vehicular Clouds to the Cloud to relieve the burden of running numerous applications and sensory services in vehicles [2]. This work advocates the prospect that 
Table 2.1: Comparison of the Related Works.

\begin{tabular}{llllll}
\hline Works & Conn & Comm & Mobility & Approach & Availability \\
\hline$[7]$ & No & V2V & High & ICN* & No \\
{$[17]$} & No & V2V & Low & SMDP & No \\
{$[34]$} & No & V2V, V2I & High & FQSS & No \\
{$[22]$} & Yes & V2V & High & P2P & No \\
{$[19]$} & No & V2V,V2I & High & clustering & No \\
{$[24]$} & Yes & V2V,V2I & High & LBD & No \\
{$[9]$} & No & V2V & High & CWS & No \\
Proposed & Yes & V2V,V2I,V2X & High & MDP & Yes \\
\hline
\end{tabular}

Vehicular Clouds will be vital for backing up IoT.

\subsection{Summary}

Several works have demonstrated the importance of mapping and modeling link stability and network connectivity for Vehicular Networks. Such works have attempted to enhance connectivity through heavily mobility-oriented approaches, where the position, speed, and acceleration of vehicles and traffic network topology are substantial elements in their models. Complementarily, we have observed steep advancements in communication technologies; for instance, cognitive radio has become a key enabling technology of dynamic spectrum access to achieve better exploitation of radio spectrum $[11,12]$. As a result, even though mobility is an important influencing factor to connectivity, urban environments are more comprehensive and contain many more opportunities for devices and vehicles to connect over multiple media, following V2V, V2I, and V2X communication methods. Provided these existing and growing alternative and redundant network connections, we propose an approach that more closely matches with the reality of urban centers and can take better advantage of cementing technologies to build and manage VCCs.

There are works, above mentioned, that related to ours in terms of VCC resource management and link stability. Table 2.1 demonstrates similarities and differences of various works and the major difference between our proposed work. The majority of the works does not include connectivity and none incorporates availability. Most works utilize the communication interface from V2V and V2I. These work mostly focused towards the mobility rather than the connectivity. The environment comprise of a urban centre; consequently, the studied scenarios relate to networks built with 
vehicles where the node density is high. Some of these works fall under resource management category. Our proposed work classify the available resources according to the reliability of their connections while delivering services in VCC. 


\section{Chapter 3}

\section{Problem Statement}

To enable resource sharing through Cloud service model in which nodes, vehicles, are interconnected and/or connected to the Internet. Vehicles, being part of a VC, can request services from other vehicles, Cloudlets, and the remote Cloud servers. On the other hand, these same vehicles can provide services to other vehicles and devices in Mobile Edge Computing paradigm, shortening the communication distance in light of decreasing service times. The largest problem in managing Vehicular Clouds resides on the fact that vehicles present high mobility, which severely compromises communication stability.

Defining the Vehicular Cloud Computing scenario in the context of urban computing allow us to precisely identify and reason the problem that is observed and tackled in this work. Thus, we understand that vehicles move around, according to a particular mobility model inherent to the characteristics of a urban centre and following the topology of urban road segments. In this dynamic scenario, connected vehicles exchange data, services, and resources through wireless communication links in a opportunistic fashion, where connections may be abruptly interrupted permanently by moving out of range or momentarily by conducting handoffs over multiple access points.

In this same scenario, we consider the existence of multiple possible communication means, such as Vehicle-to-Vehicle (V2V), Vehicle-to-Infrastructure (V2I), and Vehicle-to-Everything (V2X). As a result, this diversity and multitude of communication opportunities directly provides a high chance for vehicles to be connected to the Internet and to each other. This assumption in this work is justified with the several technological advancements that made feasible a realistic context where many communication media in urban centres contribute to keep vehicles connected. The connectivity chance is then much higher when compared with the scenarios in past 


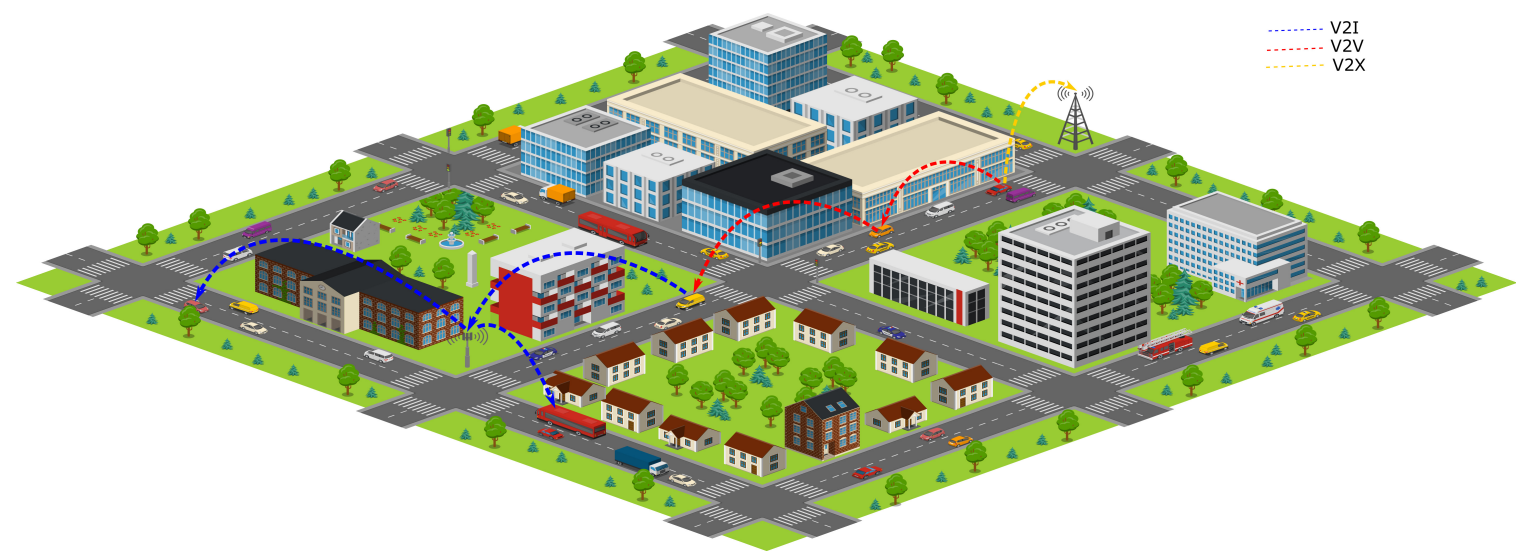

Figure 3.1: Vehicular Clouds in an urban scenario.

works where V2V communication was assumed the predominant method for vehicles to communicate.

Therefore, a general possible Vehicular Cloud scenario is depicted in Figure 3.1. The figure illustrates a single vehicle connecting through V2V communication and through LTE in the dynamic context of a urban environment where vehicles are moving and supporting the connection of the vehicle through multihop message passing protocols. Vehicular Clouds can be built through 1 or more sets of vehicles $V=\left\{v_{1}, . ., v_{m}\right\}, m \leq n$, where $m$ is the maximum number of vehicles that might compose a Vehicular Cloud and $n$ is the number of vehicles existing in the area. Such vehicles move around a urban area, following certain traffic network topology, where road segments are edges and intersections are vertices. RSUs, $R=\left\{r_{i}, . ., r_{p}\right\}$, are deployed over the urban area; these RSUs, besides their role in the traffic management system, provide certain level of coverage to access the V2I. As realistically expected, the area can present some cellular communication towers $L=l_{1}, . ., l_{q}$, which, similarl to the RSUs, are deployed over the area. Vehicles may enter and leave the urban region. These vehicles can connect with each other, defining a V2V link $c_{v_{i} v_{j}}$. We can also have vehicles establishing a link with RSUs, $c_{v_{i} r_{h}}$, and with cellular towers, $c_{v_{i} l_{k}}$.

When placed in such more favorable connectivity scenario, past VC resource management were supported through cluster-based approaches that over-stress the control to keep the consistency of the highly-mobile distributed system state. This type of control ends up requiring constant and frequent monitoring of vehicles to maintain a one-hop range micro Cloud. The management makes heavy use of mobility models to estimate the position of vehicles and increase the possibilities in which a vehicle can be reachable. This micro-management thus demands considerable efforts in tracking nearby vehicles and organizing cluster dynamically, which may render a 
high number of false positives due to the high mobility of vehicles and their movement unpredictability. Generally, assume the expected in-range communication time between two vehicles, $\mathbb{E}\left[C_{r_{i j}}\right]$, as inversely proportional to the relative velocity between the two, $\left\|\overline{v_{i}}-\overline{v_{j}}\right\|$, and dependent on their current position. In simpler terms, $c_{r_{i j}}=r /\left\|\overline{v_{i}}-\overline{v_{j}}\right\|$, where $r$ is the communication radius of the VCC cluster head.

The distribution of vehicle speeds directly conditions the prospects of successfully assembling such Clouds and making them capable of fortunately responding to service requests. At the utmost optimistic scenario where all vehicles within a VC move together at the same speed, such as in a platoon, their group movement also forces frequent changes of point attachments to the Internet or points of contact with nodes external to the platoon. The general case eventually induces highly frequent updates due to members leaving or entering a VC or cluster heads loosing contact. However, in realistic scenarios where vehicle speeds and trajectories are different, vehicles will reside within a VC momentarily, requiring frequent monitoring and adjustments; for instance, assuming communication radius of $300 \mathrm{~m}$, speed range of $10 \mathrm{~m} / \mathrm{s}$, multi-lane road segment, and no change of trajectories, we may have an average residence time of $60 \mathrm{~s}$.

Moreover, Edge Computing stands on shortening the distance between services and devices/requesters. However, the distance relates to the network distance for the sake of decreasing, or minimizing, communication latency and overhead. Therefore, we consider, or understand, that the fundamental factor for determining a vehicle as part of a Vehicular Cloud is the connectivity of the vehicle and not its geographical position and direction of movement. However, the geographical position of nodes that rely on wireless networks to be connected benefit their communication conditions, but the physical proximity of nodes may not directly define the quality of their inter-connectivity. As far as a vehicle can be connected and reachable, with certain guarantees, it can exchange services and resources with any "requester", which could be other vehicles or mobile devices.

The challenge in this relaxed case consists of precisely determining, or estimating, which vehicles are more suitable for fulfilling service requests based on its "connectivity status". A connectivity model is sought in this work in order for abstracting the intricate, inherent mobility idiosyncrasies. Availability is then the essential factor in classifying and indexing vehicles that may be participating in the "Cloud". Modeling the possible connections allows us to set a first step towards determining, with certain degree of confidence, reachability and suitability of service provider to requests. 


\section{Chapter 4}

\section{MDP-based Connectivity Modeling}

The model introduced in this work is intended to represent, and estimate, the likelihood in which a vehicle may have its connectivity status kept or changed. Through this MDP-based model, we represent the connection status uncertainty from the mobility. Optimistically, it provides the best future case scenario where vehicles may face. This optimal case can be accounted for defining which of the available vehicles are more suitable for serving requests over time. It extends the traditional resource provisioning models where just capabilities and communication delays are usually considered for the decision-making when pair-matching a requester against a set of providers.

\subsection{Model}

We assume that the behavior of vehicles in the sense of their connectivity patterns is modeled either individually or collectively, following the uncertainty from their mobility patterns and the wireless signal propagation and attenuation of the environment. To model the uncertainty probabilistically, a Markovian Decision Process (MDP) is employed, which is defined following a 5-tuple definition $\operatorname{Conn}_{i}=\left(\mathbb{X}_{i}, \mathbb{A}_{i}, P_{a_{i}}, \mathbb{R}_{a_{i}}, \gamma_{i}\right)$. In a more "relaxed" fashion, the model maps the opportunities a vehicle may have to connect over a set of heterogeneous media, involving vertical and horizontal hand-offs, as well as interleaving connections.

Note that we assume in this proposed model that the underlying communication system supports the model with relatively recent status data; media and messages exchanges allow the monitoring of communication conditions. Such monitoring metrics are presumed to be locally fed in the model, constantly, for representing the most recent communication status of a node, vehicle. Vehicles may also resort to periodic 
Beaconing for probing communication conditions.

\subsubsection{State Space}

Each vehicle may be connected through different means, including multiple means simultaneously. For instance, a vehicles may be connected to the "Internet" through other vehicles or through an RSU. Each communication mean represents a connectivity link that enhances the reachability and network throughput of a vehicle. Each of these connectivity opportunities can be mapped into states, including the overlapping connection situations. In other words, the connection of a vehicle can transit among states which allow us to produce the all possible connection combinations in the most optimistic scenario over a given number of communication means, $n_{c}$. The number

of combined states can then be cumulatively inferred as $2^{n_{c}}-1\left(\sum_{0 \leq k \leq n_{c}}\left(\begin{array}{c}n_{c} \\ k\end{array}\right)\right)$; if we include the state where there is no connection at all, the model has then $2^{n_{c}}$ states. We can also assume that this set of connectivity states composed a fully connected directed graph, where there is always a transition leading from state $s_{i}$ to state $s_{j}$. In such graph at its simplest, there are $\left(2^{n_{c}}\right)^{2}$ edges, or state transitions.

For instance, in a real scenario, we envision that vehicles may be simultaneously connect with RSUs, other vehicles, and LTE towers $\left(n_{c}=3\right)$. Moreover, in the peculiar case of $\mathrm{V} 2 \mathrm{~V}$ connections, a vehicle observes a multihop, on or more hops, connection. However, mapping all possible multihop combination scenarios in the model leads to exponential growth in this discrete State Space. Thus, accounting simplicity, we summarize the multihop V2V connection to a singular state factor, in which the number of hops directly impacts connection stability and bandwidth; this impact can be either measured through end-to-end checks or estimated over time periods. As a result, this scenario allows us a set of 8 states with 64 possible distinct transitions.

\subsubsection{Action Space}

The actions related to gaining or loosing connectivity as vehicles move around an urban area. As a result, the actions are grouped in two sets, "connecting" or "disconnecting" from certain media. For instance, in a scenario where all possible communication media is through another vehicle or an RSU, we have as actions the following: connecting to vehicle, connecting to RSU, disconnecting from vehicle, and disconnecting from RSU. The uniqueness of the vehicular network scenario grants the representation of transition from one state to another through a single action, 
such as the action "disconnecting from vehicle" leads the transition from the state "connected to RSU and vehicle" to "connected to RSU". Assuming an $n_{c}$ number of media available, we will have an Action Space $\mathbb{A}_{i}$ of $2 * n_{c}$ possible actions.

However, the action space is reduced when considering the restrictive transition of communication conditions. Thus, given an state $x_{i}$ of vehicle $i$, there is a set $\mathbb{A}_{i}(x) \subseteq \mathbb{A}_{i}$ of available actions that lead to states where a single transited state is $x_{i} \in \mathbb{X}_{i}$

\subsubsection{Transition Probabilities}

Employing a randomized model, for each action $A_{i}(x)$, there is a transition probability on $x$. These probabilities are assumed to represent the chances the connection status may change, being conditioned through a recent past. In the model, the transition probabilities may be assigned or adjusted through statistical analysis, such as time series, following recorded past transitions.

Since we assume that transition probability from any state leads to $p(\mathbb{X} \mid x, a)=1$, we can formulate as a starting model set as equal transition probabilities in the situation that there is no defined probabilities - initial values when starting the model. This starting setup is described in Equation 4.1. Then, for instance, each individual vehicle can update these probabilities independently to express their current behavior accurately.

$$
P_{x_{i}, x_{j}}(a)=\left(\begin{array}{ccc}
1 / 2^{n_{c}} & \cdots & 1 / 2^{n_{c}} \\
\vdots & \ddots & \vdots \\
1 / 2^{n_{c}} & \cdots & 1 / 2^{n_{c}}
\end{array}\right)
$$

\subsubsection{Rewards}

In each model state, there is a reward that represents the connectivity quality of a vehicle. Thus, an one-step reward is defined as $r\left(x_{i}, a_{i}\right)$, denoting the reward using action $a$ in state $x$. Quality can be a cumulative value originated from several factors, such as link stability and end-to-end bandwidth, where both might be projected over time. In the proposed approach, rewards directly indicate the best possible opportunities in terms of network reachability. 


\subsubsection{Policies}

The Markov policy $\pi$ of this model is, by definition, stationary and randomized since $\pi_{n_{c}}$ does not depend on $n_{n_{c}}$, and the selection of the actions follows a probability distribution that generally produces a $p\left(\cdot \mid x_{i}, a_{i}\right)$. By definition, a policy corresponds to a sequence of transition probabilities $\pi_{n}\left(a_{n} \mid h_{n}\right)$ from a n-step history $H_{n}$ to $\mathbb{A}$, in given number of iterations $n \in \mathbb{N}$. Again, policy $\pi$ is characterized by a transition probability $\pi$ that relates to $x \in \mathbb{X}$ mapped to a $a \in \mathbb{A}$ where we summarize as $\pi(\mathbb{A}(x) \mid x)=1$ for all $x \in \mathbb{X}$.

From the model, we can obtain an optimal policy that corresponds to the "best" actions to maximize rewards in light of the conditioning transition probabilities. Therefore, this policy is employed as an indicator that optimistically estimates the communication conditions with vehicles. All vehicles show these optimistic estimates as a baseline that is used to classify them against service requirements.

\subsubsection{Transition Discounts}

Discounts are applied to weigh down future steps/iterations in relation to the current state $x_{i}$ where the connection status is. To deal with expected total discounted reward, the discount is defined as a fixed value in this model as $\gamma \in[0,1[$. We express the expected total reward over the first $\mathrm{n}$ steps, $n \in \mathbb{N}$ as in Equation 4.2.

$$
v_{N}(x, \pi, \gamma)=\mathbb{E}_{x}^{\pi}\left[\sum_{n=0}^{N} \gamma^{n} r\left(x_{n}, a_{n}\right)\right]
$$

In other words, we define the value function $v(\mathbb{X})$ as the sum of all predicted future rewards, implementing temporal discounting with a gamma parameter where rewards are at $k$ steps in the future are weighted by an exponential discount factor $\gamma^{k}$. The value function turns in a weighted sum, described in Equation 4.2.

\subsection{Connectivity Estimation}

Assuming an infinite horizon when utilizing the reward function, described in Equation 4.2, we employ Bellman Equation [31] to define an state value function in state $x \in \mathbb{X}$ for a stationary policy $\pi$, as described in Equation 4.3.

$$
V^{\pi}(\mathbb{X})=r(x, \pi(x))+\gamma \sum_{y} p(y \mid x, \pi(x)) V^{\pi}(y)
$$




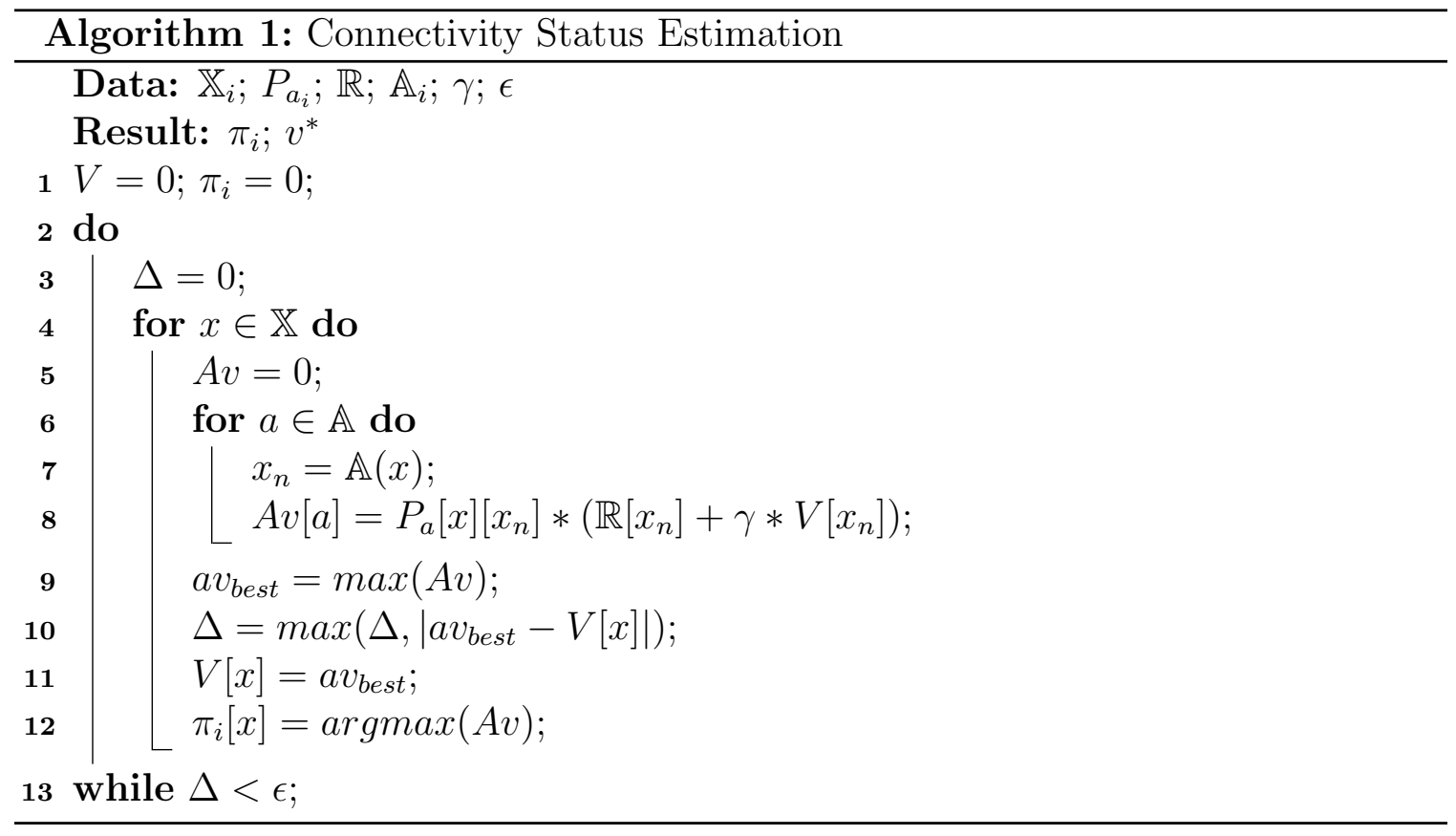

Following the Principle of Optimality of Bellman [31], we establish an inductive greedy search process which takes as basis on an initial state and decision, identifying an optimal policy in the subsequent decisions/actions. Thus, the principle of optimality applied over the value function gives the optimal value function $V *=\max _{\pi} V^{\pi}$, represented in Equation 4.4.

$$
V^{*}(x)=\max _{a \in \mathbb{A}}\left[r(x, a)+\gamma \sum_{y} p(y \mid x, a) V^{*}(y)\right]
$$

Defined in Equation 4.5, the optimal policy from a given state $x$ follows a similar representation of the optimal value function.

$$
\pi^{*}(x)=\underset{a \in \mathbb{A}}{\arg \max }\left[r(x, a)+\gamma \sum_{y} p(y \mid x, a) V^{*}(y)\right]
$$

Equations 4.4 and 4.5 can then be translated to a Value-iteration algorithm where it iteratively searches for the best policy given transition and reward matrices. The value-iteration search is summarized in Algorithm 1. According to the algorithm the search ends when an error $\epsilon$ is satisfied. Both discount $\gamma$ and error $\epsilon$ condition the convergence and number of $k$ iterations in which the algorithm runs, $k=\frac{\log \left(r_{\max } / \epsilon\right)}{\log (1 / \gamma)}$. The processing to identify the current suitable pseudo-optimal MDP policy of our model is conducted for each vehicle. 


\subsection{VC Management}

The Vehicular Cloud Management then fundamentally employs the proposed model in order to differentiate vehicles based on the quality and stability of their point of attachment to the network. The management can follow many architectures where centralized, hierarchical, or fully distributed service orchestrators allocated resources dynamically. In our envisioned scenario, the a set of VC Management Units serves as reference points in which nodes, mobile devices, pose service requests that match up with surrounding (in terms of network) vehicles. The whole scheme works in 3 phases: monitoring, calculation, and advertisement. In fully distributed fashion, Vehicles monitoring their own current connections and update their MDP transition and reward matrices, individually. The optimal policy search algorithm can be executed on event basis or periodically.

In this work implementation, we employed a event-oriented triggering of the search whenever an update occurs on the matrices. The connectivity usually relates to a reference point, which is the point of attachment in this work. Thus, due to this assumption, this point of attachment, which can be an RSU, tower, or VC Management Unit, also becomes a reference for devices and other vehicles to contact and find service providers. Vehicles notify their respective management unit, which then classifies them accordingly. The unit finally matches incoming service requesters based on the ranking it updated. 


\section{Chapter 5}

\section{Connection Cycling \& Pattern Modelling}

Intrinsically, the MDP-based connectivity model relies on vehicles repeating connectivity patterns. If vehicles do not revisit connectivity patterns, the model potentially fails. Vehicles will connect to the RSU using any of the such states, V2V, V2R, V2L, those represents connected state or !C, which represents disconnected state. But for our purposes, reducing these states into two states, connected or disconnected. This simplify the problem and allows for additional metricmodel to play a part in proving the MDP-model results. The metric applying to determine the result of MDP-model is known as, availability. Availability is probability of service being online for duration of the interval. The definition of the availability metric changes based on the area or based on the proposed solution for the problem. There is disparity between cloud provider and academic research. Even within the cloud provider, there are various ways of calculating the availability metric.

\subsection{Availability}

Observing the communication patterns from the mobility of vehicles, we could match them to a reduced availability model: vehicles being either connected or disconnected. A availability is a concept extensively considered for countering faults in systems and enhancing their fault tolerance. Availability provides a probability where the system or systems are shutoff from the service. The availability is used for calculating how long does the system will stay up or in service. This metric gives us the probability of system during the interval of which the services are available. This metric is useful for understanding how systems interact or provides services and how long they generally 
stay up. There is quite a bit of disparity on how this metric is calculate between the cloud providers and in the academic research, but the general concept across the cloud providers and in academic area stays the same [26].

$$
A=\frac{M T T F}{M T T F+M T T R}
$$

\subsubsection{Definition of Availability}

There are two areas where the different definition of the availability comes into play. The cloud providers, such as Amazon, Google, Windows Azure, and Rackspace, have different perspective on availability. Amazon calculates the availability based on the services chosen from the client. For instance, web services from the 100 percents subtracts the percentage of shutdown minutes which provides the availability of that service [26] and that just for one of its services. Other services perform different calculation to determine availability. Google calculates the availability using Equation 5.1, where MTTF: Mean time to Failure and MTTR: Mean time to Repair, but it only takes downtime if the service is down for a minimum of five consecutive minutes [26]. Microsoft has similar idea as Amazon, but it calculates the uptimes of the services monthly, and the downtime is calculate only when there are more than two servers are down in different update domains and fault domains [26]. Lastly, Rackspace does not consider maintenances and emergency situation as the downtown and because of that reason they provide almost 99.9 to 100 percent availability guaranteed for there services [26]. There are just most common services provided by these companies, there are different level these services and one can go find more information by either reading the Service level agreement (SLA) or the survey work on availability of Cloud providers [26].

Another type of availability definition drives from the research in academic areas.

Even in this area, there are multiple ways availability can be calculated. In the academic area, the general concept and equation stays the same. Even most of the researchers use similar equations that driven from Equation 5.1. Some works grabs availability definitions from the SLAs, and some other works redefine availability to fit there proposed solution [26]. In some works, the definition of the availability is linked directly to the performance parameters of the system and the response time of the system. By determining if the response time of the service is fast or slow, they can determine the performance of the web service. This leads to applying availability model to the service response time, if the response time of the service is fast, then 
that classify as high availability. If the service time is slow, then that degrades the performance of the service [14].

The common way to measure availability in the cloud is applying Equation 5.1 on the service and that acquires the probability, which is presented in range of $0 \%$ to $100 \%$. Usually, achieving the $99.99 \%$ availability shows, that the given service has a better sustainability or stays on for most of the time in a year. The closer probability is to 1 , the higher sustainability this service provide. As mentioned in previously, most of cloud providers use probability to measure how available there service is. This approach is also known as four nines or five nines. The higher the nines are, the better availability of the service [26]. In this work, five nines seems to be just a standard that varies among the cloud providers, no research-supported work has employed such a metric for classification or decision-making. There is extensive discussion about five nines in the SLA.

Service provider thus has its own table to measure what exactly five nines metric measures. The general consensus is that $99.999 \%$, which is five nines, represents approximately 5 minutes of downtime in the year and that is for the maintenance of the service. For example, Amazon SLA shows table describing 'five nines' metric calculation of there services [30]. Mircosoft describe five nines metric for each of there services in a detailed manner ${ }^{1}$. Furthermore, Google has provide SLA for its service as well ${ }^{2}$. The most common aspect of using monthly uptime to calculate the five nines for each of the companies. The main difference is the parameters adopt for the metric calculation. For example, Google, "Downtime" means that the error rate parameter is more than 10\% (Google) and "Downtime period", if the customer application is down for consecutive minutes (Google). In comparison to mircosoft, where parameters just depends on the service itself. Suppose the customer chose Azure Bot Services, the "Downtime" represents if the HTTP request is not reached to the customer (Microsoft), the definition changes per service. Each service provider produce detail description in SLAs for customer to understand the parameters involved in the calculation of 'five nine' metric.

\subsubsection{Availability and Connectivity}

The definition of availability for this work is similar to the definition mentioned in the pervious section, but modified to fit our needs. Nevertheless, general concept

\footnotetext{
${ }^{1}$ Microsoft. Service Level Agreements. https://azure.microsoft.com/enus/support/legal/sla/?cdn=disable, 2020/01/14

${ }^{2}$ Google. Service Level Agreements. https://cloud.google.com/appengine/sla, 2020/01/14
} 
of the availability will stay the same. We still applying Equation 5.1 to obtain the probability of the resources that are connected. Our definition of the availability has to adopt the general concept but differ from the definitions mentioned previously. Because, the nature of the problem is vastly dissimilar to cloud problems. Firstly, the context of our problem is of a vehicular networks and the concept of uptime and downtime is incompatible in comparison to the cloud services. The concept of uptime and downtime in vehicular network can be mapped to connected or disconnected. State of the vehicles changes from v2v, v2r or v2l, rather analyzing pattern in these states, a simplification of only two states: connected or disconnected reduces the problem, where availability model proves to be convenient. Secondly, there have not been enough works that applies availability in same problem as ours .

Mostly, availability is calculating ratio of service being online for duration of the interval. In our case, vehicles have states that are evaluated by the MDP-model and given a value to the vehicle, that determines if vehicle is good resource to utilize. Since, all the network is based on the connectivity with the vehicles and RSU, which is new paradigm and very specific niche. Furthermore, applying availability model to check the repeatability of the states of the vehicle, this work dives deeper into this specific niche, that as of now lack the research. Most research works that are based on the availability model are in the mobile cloud computing or in the distributed system as shown in this paper [26].

Lastly, most of the resources are mobile, which drastically modifies the definition of availability in this problem. Since, vehicles have high mobility and have different states that are analyzed by the MDP-model, but does not have either uptime or downtime of the vehicle. Impartial from the cloud in distributed systems, vehicles do not just shutoff, they could travel further away from the range of the RSU and that causes them to be disconnected or move within the range of RSU or other vehicles that are close to the RSU, which results in being connected. This could repeat multiple times and produces a pattern during the simulation, that arises the use of availability model in which uptime represents connected and downtime represents disconnected.

Generally, availability has uptime and downtime. Uptime being how much of the portion the services has been active during the duration of the interval. Downtime is opposite of uptime; it is the time that services have been inactive for the duration of the interval. For this problem, uptime is switched to how many times resources connected and stay connected to the RSU and downtime how many time did resources disconnect from the RSU. Employing this definition, we can find the probability of availability for each resources within the network. This metric develops a deeper 
understanding of how MDP-model evaluates these resources and assign the value. For example, in the results, we notice that the MDP-model nominates higher value to nodes that stay connected to the RSU often comparing to the nodes that do not. Furthermore, availability helps evaluate results the MDP-model. If the MDPmodel elect higher value for vehicles, that should culminate in more connection than disconnection or no unavailability of the vehicles over the duration of the simulation.

\subsubsection{Taxonomy of Availability}

Taxonomy can help contextualize availability model into the scope of this work. There are two concepts that are core topics of this work: Connection repeatability and Connectivity Cycling Patterns. Connection repeatability take place at the communication level, where vehicles have states that repeatability connect to the RSU, that causes a pattern to form over the duration of the simulation. This leads toward Connectivity Cycling Patterns, each vehicles suppose to have a patterns, that pattern determines the level of connectivity. If the patterns repeats, the MDP-model notice the connection to be constant with the RSU. Then primary focus becomes the cycles, that are manifest from the repeatability.

\section{Detection Interval}

The mobility of the vehicles plays important role in determine the unavailability or disconnection that vehicles would face over the interval or duration of the simulation. Disconnection should decrease value from MDP-model, since more unavailability of the vehicles will prove to be worse resource within the network. There are some metrics covered by this taxonomy, that are utilized to determine the availability of the resources. First, its MTTF: Mean time to failure, in this scope known as uptime, which represents that resource is connected to the RSU. Second, its MTTR: Mean time to Repair, in scope of this work, known as downtime, which represents that resource is disconnected from the RSU. Lastly, by monitoring these metrics and combining them to figure out the availability of the resources as shown in Equation 5.1. Figure 5.1 presents the two types of the detections. The MTTF (uptime or connected) and MTTR (downtime or disconnected).

\section{Unavailability or Disconnection}

The communication between the vehicles and RSU happens through the V2R (Vehicleto-RSU), V2V (Vehicle-to-Vehicle), V2L (Vehicle-to-LTE) or !C (not-connected). Ve- 


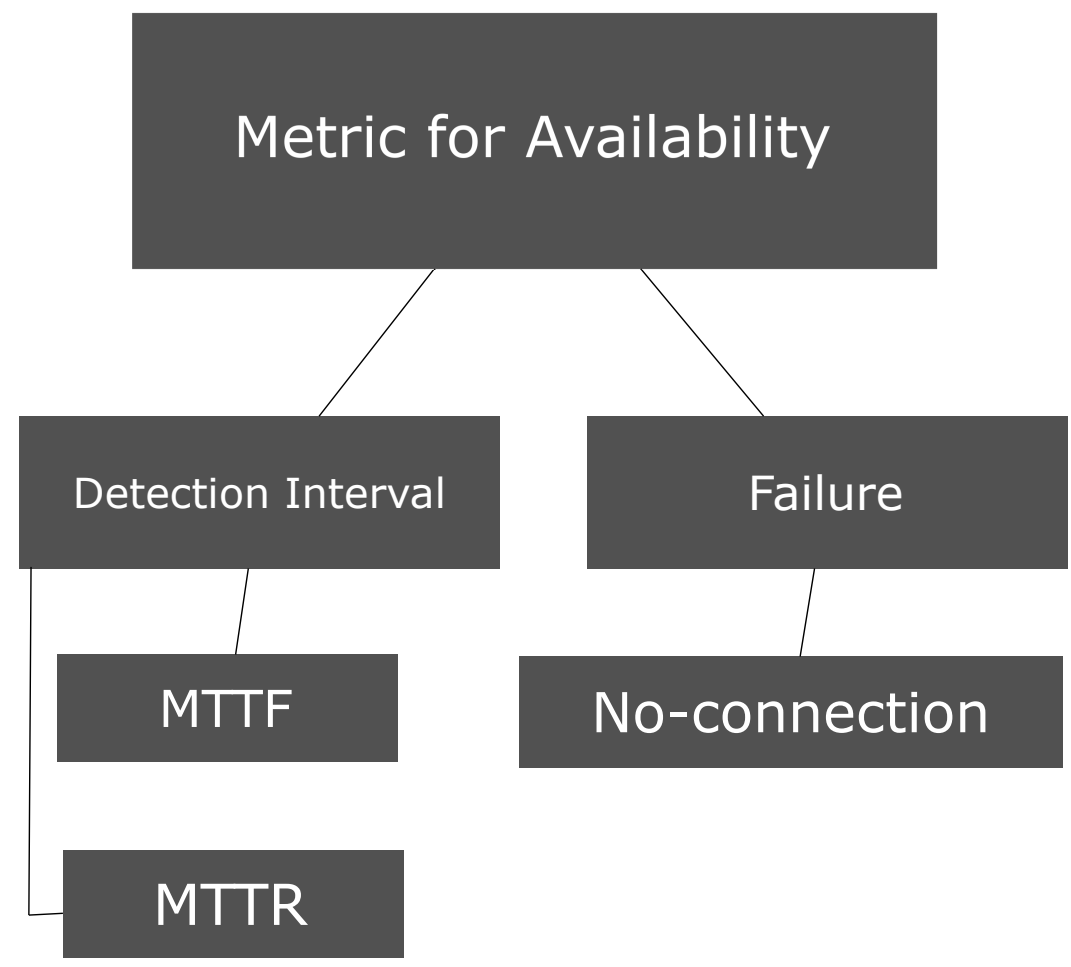

Figure 5.1: Availability Taxonomy.

hicles in mobility may switch between established forms of communication, this may result in disconnections if vehicles moves further away from range of RSU and even through the hops is not possible to reach RSU. This is possible to detect through the periodic beacon messages from the RSU. As these beacon message travel floods the network, vehicles reply back only if they are within the range of the RSU, LTE tower, or if beacon message reach through hops before message expire. If node is moved out of the simulation completely, then the state transit to the !C and that resource disconnected for the rest of the simulation, as no reply will be received from this vehicle. Another scenario involves a node moving out the range of RSU, but since there is multi-hop option, the node could still be connected through V2V or V2L. The only way this node could not be reached its if its on the isolated and there is no other nodes around it or the number of hops is far exceed for required communication to happen. In figure 5.1, sub-category of failures shows no connection has been initiated from the nodes, that exemplifies the poorest scenario as the MDP-model becomes ineffectual. 


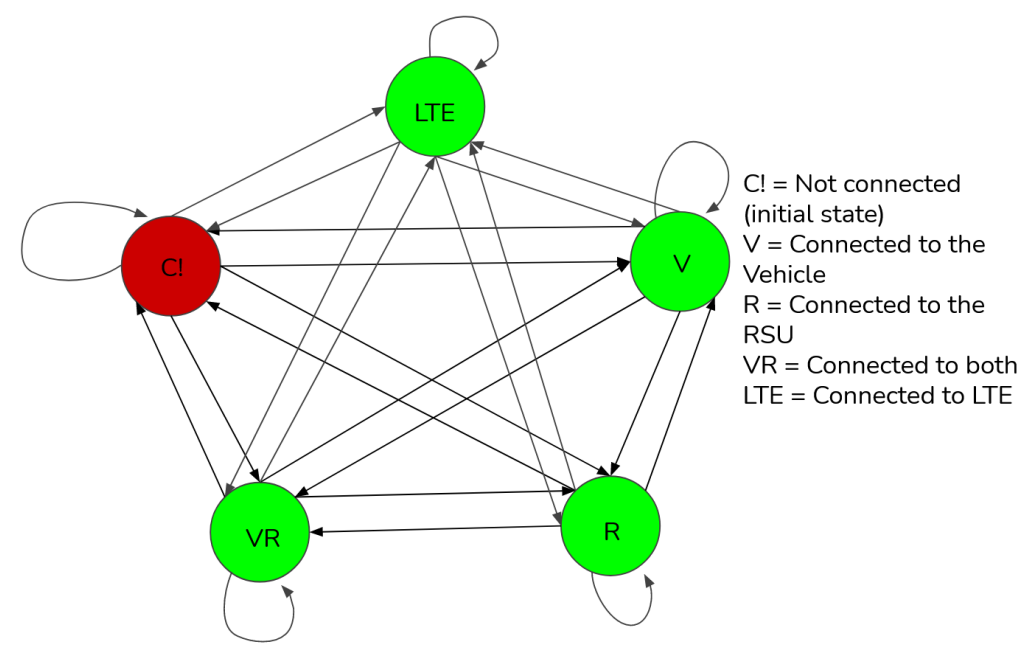

Figure 5.2: State \& Transition of the Resources.

\subsubsection{Cycles}

Every $\mathrm{x}$ seconds beacon messages flood the network and cycle consists of every $\mathrm{n}$ periodic beacons messages. This interval affects how the MDP-model and availabilitymodel evaluates the nodes. The periodic beacon message and length of the cycles play important role in determine the availability of each vehicles. Suppose periodic beacon messages happen quite often arising more communication of vehicles with RSU strengthening connections providing more accurate availability, but there is downside to this as well, this require to flood the network and slow down the simulation and causes more collision within the network as well. Suppose the cycles happens quite often, resulting in more disconnections as nodes in high mobility may move out of the range, causes the state of the node to transit into not connected, increasing disconnected, but it might come within the range in next cycle.

\subsubsection{Patterns}

The connectivity of the vehicles issues repeated states that form a pattern. Now, there is a couple of ways to monitor these pattern. Reducing the pattern to simple true or false parameter associate with each node connection. When the node is connected to the RSU, then increment that variable and keep track of the count, else if the node is not connected, then another variable that keep track of the connection not made for the duration of simulation. Another way is to calculate the availability of the node with respect to the RSU. For this implemented Quality of OS application: Availability. This basically gives the fraction of time the system will be available in 


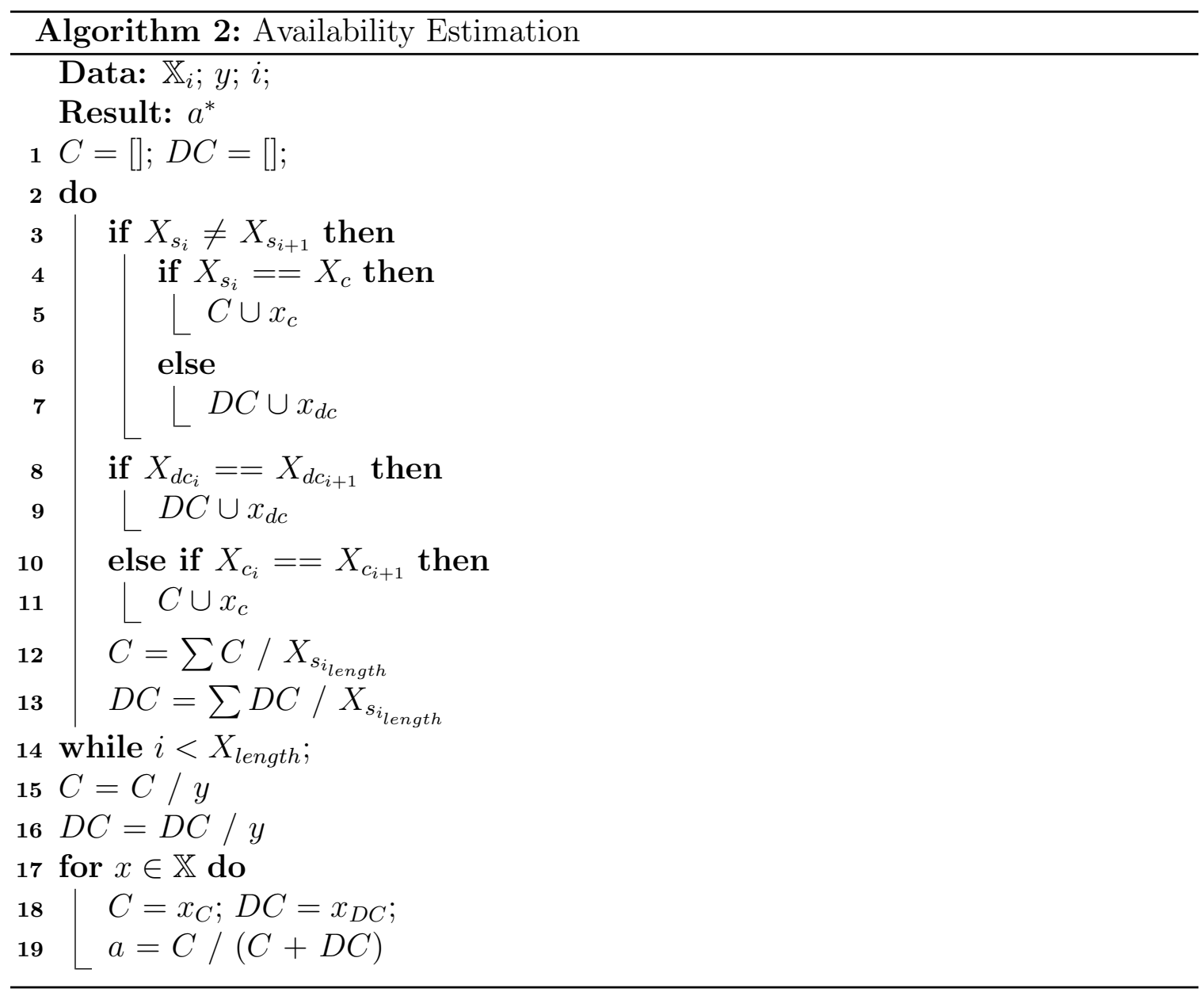

terms of the definition in Equation 5.1. The parameters are defined as follows, MTTF: Mean time to failure [26], basically, also known as uptime or connected in the scope of this work. We calculate MTTF by watching the how long the connection was stable with RSU over the duration of the cycle and all through the simulation. MTTR: Mean time to repair [26], also known as downtime or disconnection. We calculate MTTR by monitoring when the connection was disconnected during the cycles and if it stays disconnected or the node has left the simulation and cannot connected at all, at that point, MDP-model will assign the value of 0 and availability-model should result in lower ratio of availability.

The availability estimation algorithm calculates the availability for individual vehicles provided the connections and disconnection in $\mathbb{X}_{i}$, which holds number of connections and disconnections per vehicles. Parameters $C$ and $D C$ represents connections and disconnections of the individual vehicles. For every element in $\mathbb{X}_{i}, a$ represents the availability is computed and attached as the properties of that element in the $\mathbb{X}_{i}$. 


\section{Chapter 6}

\section{Performance Analysis}

We have conducted simulation experimental analysis to evaluate the performance of the proposed connectivity-oriented model. The simulations aimed to represent realistic urban center scenarios, requiring simulation of communication, as well as the mobility, of vehicles in a targeted intelligent transportation environment. In this section, we dive into Scenario used in our experiments, different types of network topology, various parameters, our way of evaluting the model, and results that are achieved by running the simulation.

\subsection{Scenario}

The experimental scenario is completely built using Veins [32], with the support of Omnetpp $++[35]$ and SUMO [18]. The simulator Veins contains general protocols and modeling capabilities of networking or wireless protocols for communication of the nodes. SUMO brings a microscopic mobility traffic simulation of the nodes in Omnet++. Veins facilitate and keeps the consistency between these two later simulators, allowing the basis for modeling and simulating Vehicular Networks.

The whole ITS scenario stands on WAVE as the supporting V2V communication means. Vehicles can "connect" to the Internet by communicating through both $\mathrm{V} 2 \mathrm{~V}$ and V2I. Thus, RSUs are also present in the simulation scenario. Relying on the IEEE 802.11p standard, the V2V communication mode follows WSMP, in which OFDM guarantees different data rates. We thus assume proper, organized one-hop communication over multiple channels through Control Channel $(\mathrm{CCH})$. 


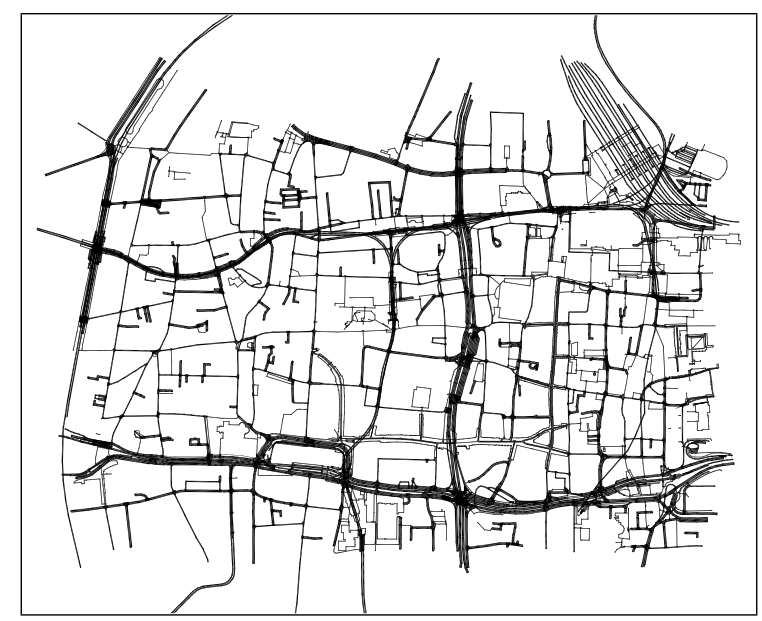

Figure 6.1: Cologne Metropolitan area used in the simulation analysis.

\subsection{Traffic Network Topology}

For the sake of realism, we use a real-world urban scenario where vehicles present highmobility displacement patterns. We use a slice of Cologne metropolitan area as our urban center, which is adopts a traffic simulation data set scenario for bringing more realistic vehicular mobility. The region represents a dense urban area of 1000x1000 $\mathrm{m}^{2}$, as depicted in Figure 6.1. Most of the map follows a standard grid layout; however, some clusters of road segments form non-conventional layouts. The urban map also shares parts with highways, allowing a wider range of speeds and mobility patterns.

The RSU is placed on top left corner of the map to maximize the number of hops achieved throughout the map. This feature is added to understand, if the model is rewarding the vehicles based on the number of hops as it is required. We also test by placing the RSU in each corner of the map and monitoring if the vehicles values changes based on the position of the RSU.

\subsection{Experiments}

We divide the experiments into two sections, because it will clarify the reasoning behind applying two different models. The first section contains MDP-model experiments, which will the grounds for Availability-model analysis. The second section will consist of the Availability-model experiments. The scenario and traffic network topology were same for the both experiments, the difference happens at the parameters, performance metrics and result sections. There are additional parameters added to the compute the availability of the vehicles and another state for MDP-model. 
The performance metric was modifiy to analysis the availability of the vehicles and the results section also updated, hence we included new subsection for each of the category mentioned. MDP-model results paved a way for the availability-model to analysis and extends on MDP-model results. The understanding of the probability of connectivity with vehicles per hop will justify the results from the MDP-model. The analysis from the availability-model will highlight the probability of the connectivity and deeper understanding how MDP-model ranking these vehicles.

\subsection{Performance Analysis of MDP-Model}

This section covers detailed description of parameters, thorough explaintation of performance metrics and comprehensive clarifictions over the results gather from the MDP-model. In vehicular networking there requires well defined parameters to configure the network, that suitable for the experiments. A specific performance metrics have been selected to explore the results obtained the MDP-model.

\subsubsection{Parameters}

To delimit and constrain our experimental scenarios, we defined combinations of parameter settings. Table 6.1 summarizes all the ranges of parameters used in our simulations.

In simulations, the speed of vehicles range between 5 and $15 \mathrm{~m} / \mathrm{s}$ to resemble to usual urban centres. Such speeds thus condition a high dynamicity in our observed scenarios where vehicles might changes their connectivity statuses quite frequently in a short span of time. Also, our scenarios adopted different densities of vehicles, which ranges from 500 to 2000 vehicles simultaneously moving in the simulated area. The density directly impacts mobility and connectivity, where sparse scenarios might lead to higher disconnection rates and high-density scenarios might allow vehicles to be connected quite often by either V2I or V2V. Observing both boundaries in the density spectrum allows us to tell if the propose model is capable to capture the respective connectivity conditions.

For this particular simulation scenarios, a single RSU is deployed for the whole urban region. Consequently, during the simulation vehicles are necessarily out of range of the $\mathrm{RSU}$, resorting to $\mathrm{V} 2 \mathrm{~V}$ communication or even being disconnected. For the simulations, the RSU is place at the bottom left quadrant of the region depicted in Figure 6.1. 
Table 6.1: Simulation Parameter Settings

\begin{tabular}{rr}
\hline Parameter & Value Range \\
\hline Urban area & $1000 \times 1000 \mathrm{~m}^{2}$ \\
Vehicle density & $500-2000$ \\
Vehicle Speed & $5-15 \mathrm{~m} / \mathrm{s}$ \\
RSU density & 1 \\
PHY model & IEEE $802.11 \mathrm{p}$ \\
Vehicle comm. range & $400 \mathrm{~m}$ \\
RSU comm. range & $400 \mathrm{~m}$ \\
Transmission power & $30 \mathrm{~mW}$ \\
$\gamma$ & $0.1,0.5,1$ \\
\hline
\end{tabular}

Besides the communication ranges adopted in our simulations, the transmission of vehicles and RSU are susceptible to propagation, attenuation, and collision issues that might occur, according to the network simulator. Such issues are expected and needed so that the proposed model can properly represent dynamic conditions while estimating connectivity statuses of vehicles.

Since the model is supposed to adapt to changes, the transition matrices are periodically adjusted according to the new communication conditions of each vehicle. At the current implementation, the RSU is the entity responsible for maintaining such matrices, where each vehicle periodically reports to the RSU its current connectivity status. The new status then drives the RSU to update transition probabilities so that they more precisely represent the connectivity behaviour of a vehicle. After some simulation time, the RSU has collected significant amount of data from vehicles, allowing it to ranking the surrounding vehicles using the proposed model.

\subsubsection{Performance Metrics}

There are two metrics in which we evaluate our model is providing the results that we intended, Ranking and Ratio. The first metric Ranking is based on the value provided by the model. The second metric Ratio is based on the connectivity of the vehicle with the RSU.

We adopted Ranking as the main a performance metric in these analyses. We rank individual vehicles based on the model value and we compare, the most "successful" and the "worst" from our ranking. The most "successful" will have constant "connectivity" to the RSU and the "worst" has no "connectivity" or little "connectivity" to the RSU. The model presents three individual states: "Disconnected", "V2V", and 
"V2I". The higher values get assigned to the vehicle, that is in the state, "V2I", which means the vehicle is connected to the RSU, and the vehicle can also maintain that connection.

The Ranking metric is used to determine which vehicle can stay in constant contact even if the hops are increased. If the vehicle stays connected to the RSU any amount of data can be transferred to the vehicle and can be retrieved as well. The model picks the best candidate for that, and this Ranking metric hence proves to be more useful. We can also compare the best top candidates with the lower worst candidates. Selecting the most "successful" candidates to distribute the data will result in the faster delivery times of the request. The situation could also change, maybe the lower candidates becomes top as vehicles get closer to RSU, which is also why we picked the ranking system as our performance metric.

The ratio of the most "successful" connections per number of vehicles is another metric observed. After being ranked, two sets of vehicles are contacted. The n vehicles with the highest ranks compose the top set while the $\mathrm{n}$ vehicles with the lowest ranks form the bottom set. We observe if the connections with the RSU persist or vehicles are reachable through $\mathrm{V} 2 \mathrm{~V}$ after they have reported their connectivity statuses through the sent messages. This metric helps establish an understanding of connectivity ratio in a high mobility environment.

\subsubsection{Results}

This section is structured in explaining the results obtained from the model. Initially, we start with initial model results, then we move to the more in depth and some optimizing parameters of the model to obtain the better performances from the model. The graphs is based on the n number of hops. Later in this analysis, we analysis more in depth of how each hop is effected and how per hop there is decreased in the value by the model.

Our metric is based on hops required to reach RSU. We group the vehicles by the number of hops, as shown in the bar graphs. We find the maximum, minimum, and the average values assigned to each vehicle by the model from each group and draw a bar graph. The y-axis represents the hops or "Reachability" and x-axis represents the maximum, minimum, and average values of the vehicle assigned by the model or in our case "Ranking". We rank the vehicles based on the value that is assigned by the model. Each group has the "best", "worst", and the "average" vehicle. In Figure $6.2 \mathrm{a}$, simulations were conducted with a discount factor of 0.1 and about 500 vehicles. 


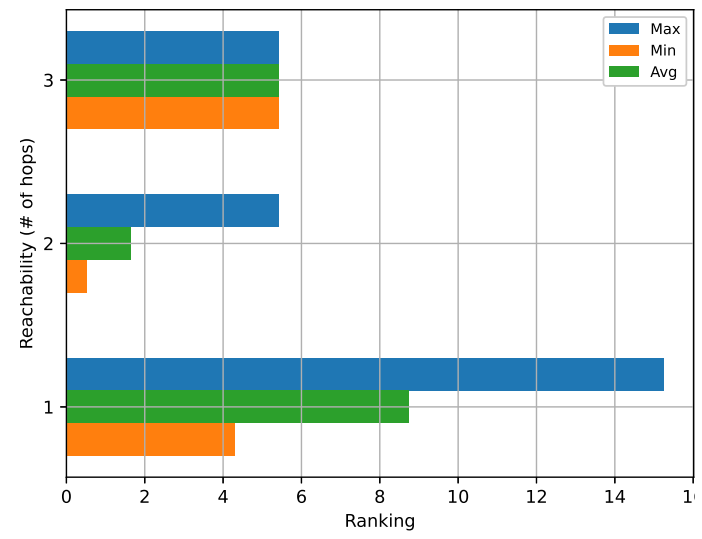

(a) $\gamma=0.1$

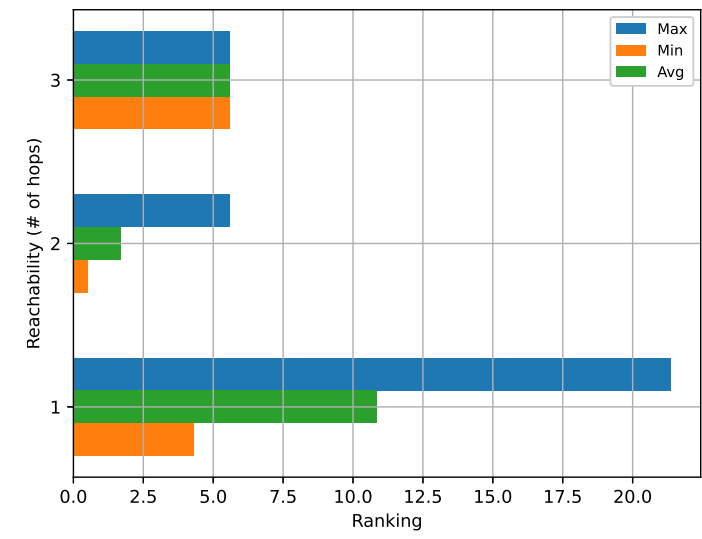

(b) $\gamma=0.5$

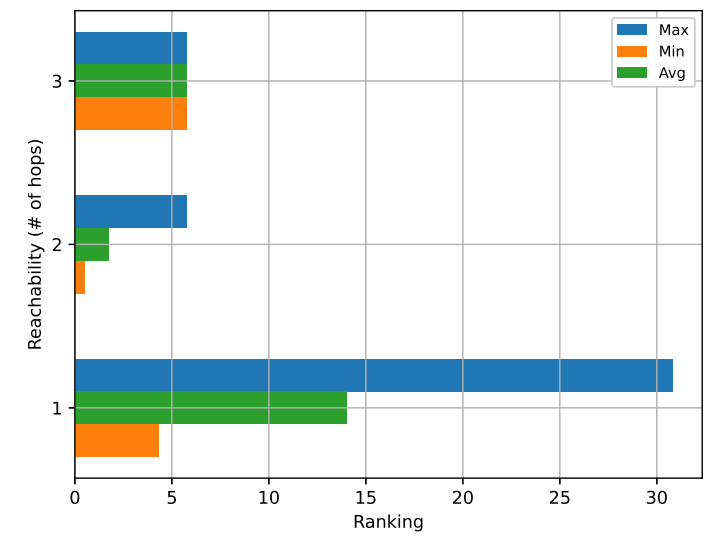

(c) $\gamma=1$

Figure 6.2: Ranking of vehicle connectivity on a 500-vehicle scenario.

The closer the vehicle to the RSU, the higher the value is going to be. The group 1 is excepted to present high values as those vehicles are only about 1 hop away from the RSU. The figure clearly shows that vehicles that are 2 hops away, named group 2 , show considerably lower connectivity status when compared with vehicles 1 hop away. Vehicles in group 2 is just slightly better then the group 3, which includes all the vehicles that are 3 hops away.

Figure $6.3 \mathrm{~b}$ has a discount factor of 0.5 and the density of the vehicles is 1000 . The bar graph here has much higher values because of the proximity of vehicles to the RSU and among themselves. The higher density in this figure thus enable a better connectivity on overall in terms of providing more opportunity to connect and stay connected with vehicles. Since, the density of the vehicles is really high, that results in much higher values by the model, as some vehicles are within the RSU range and potentially could stay within the RSU for some period of time, that makes the model 


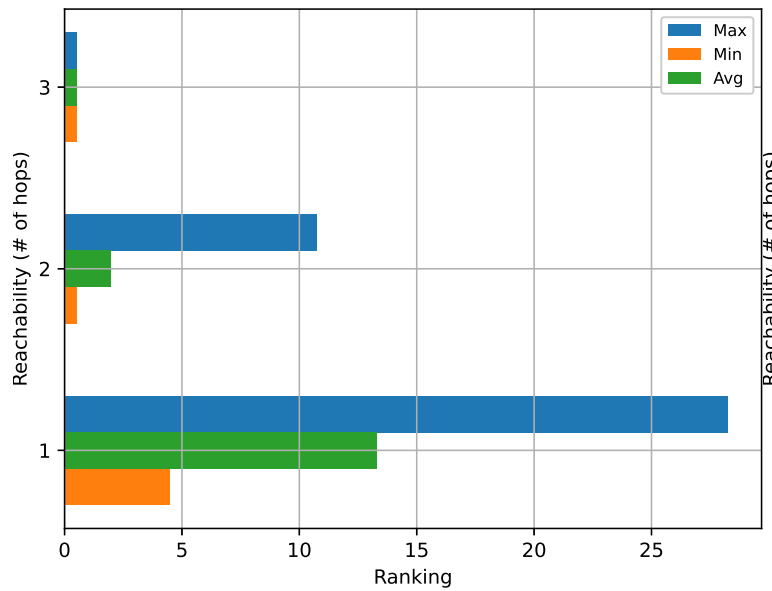

(a) $\gamma=0.1$

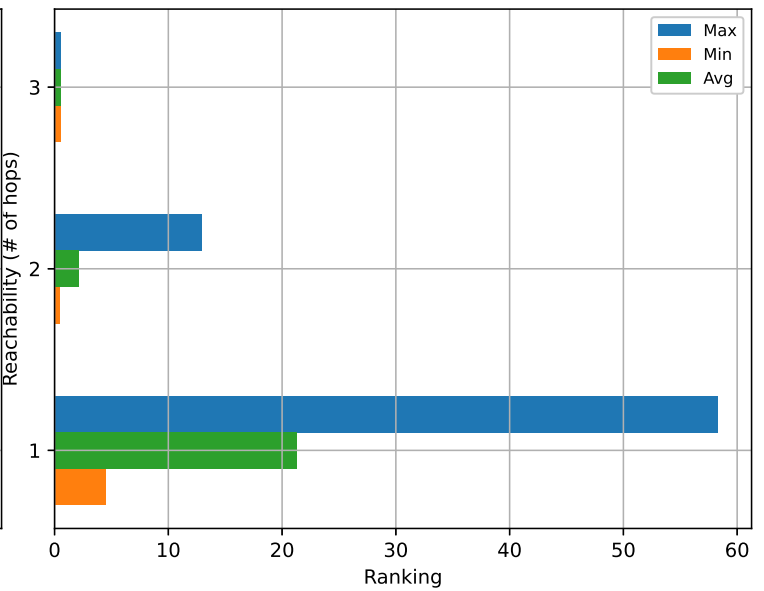

(b) $\gamma=0.5$

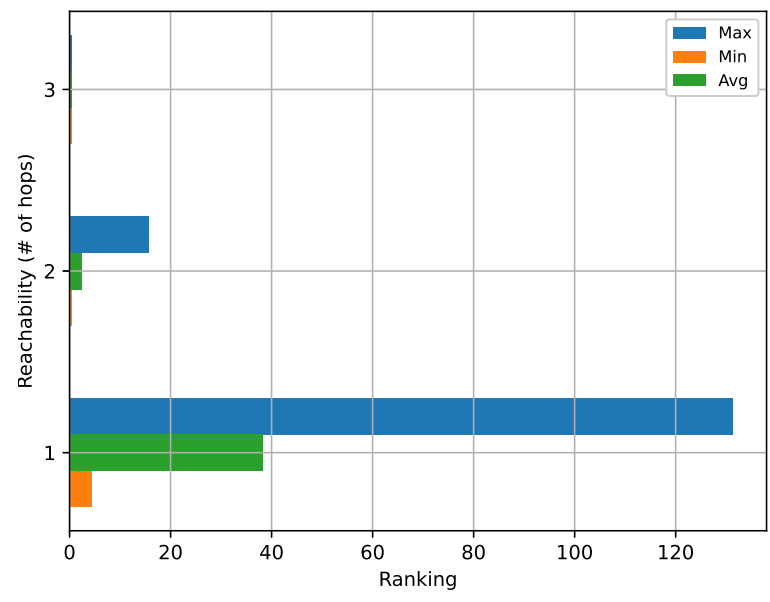

(c) $\gamma=1$

Figure 6.3: Ranking of vehicle connectivity on a 1000-vehicle scenario.

assign the values higher as the connection with the vehicle stays alive. Also, the vehicles that are constantly in "connectivity" state to the RSU also receive a higher value. This behaviour is expected and shown in both Figures $6.3 \mathrm{~b}$ and 6.2c.

We also compare the density and how it affects the ranking metric. Each of the figures are consisting of discount factor and the density of the vehicles. As the density seems to be increasing, so does the group 1 increase in the numbers and maximum values. There is a possibility the initial vehicle was 2 hops away and as it gets closer to the RSU, changing its network distance to only 1 hop away. Consequently, the values of this vehicle increases. Vehicles are always moving around; as they either get close or far away from the RSU, the model assign the value and based on the ranking. Thus, vehicles might be selected as the "successful" candidate.

Figure $6.4 \mathrm{a}, 6.4 \mathrm{~b}$, and $6.4 \mathrm{c}$ with a discount factor of $0.1,0.5$, and 1 all shows 


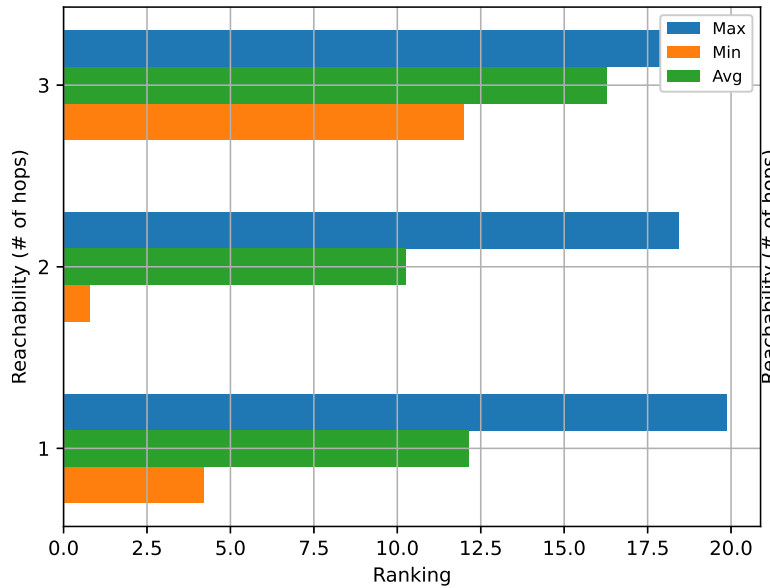

(a) $\gamma=0.1$

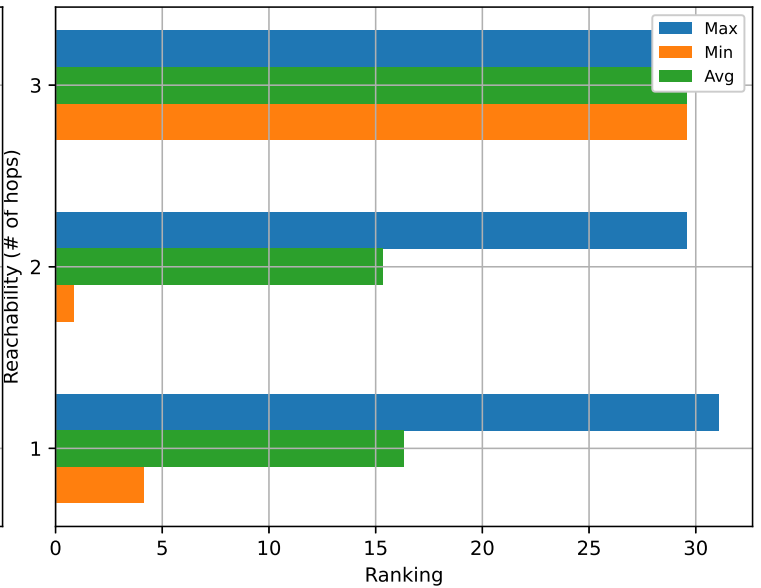

(b) $\gamma=0.5$

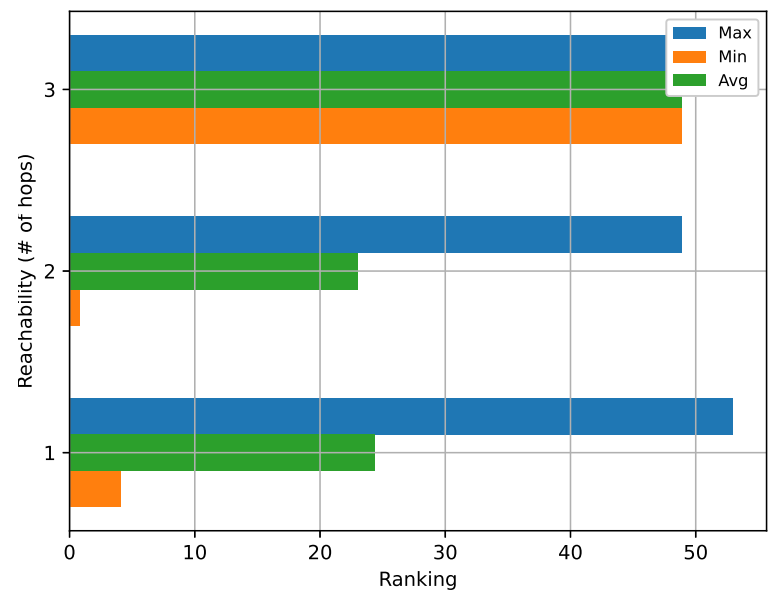

(c) $\gamma=1$

Figure 6.4: Ranking of vehicle connectivity on a 2000-vehicle scenario.

very similar values, density is 2000 . This could be anomaly but comparing to other graphs its more stable in terms of the values. All the 3-hops vehicle do not seem to move as much as 1 hop and 2 hops vehicles. They attend to stay with the 3 hops and that cause model to assign similar values regardless of the discount factor. These are earlier experiments with the model, later in this analysis as we changed the topology of the map and made some adjustment with the model provides much more improved results. That shows how model behaved in different topologies, as the adjustments with model were the parameters changes based on the topology of the map.

We also compare the density and how it affects the ranking metric. Each of the figures are consisting of discount factor and the density of the vehicles. As the density seems to be increasing, so does the group 1 increase in the numbers and maximum values. There is a possibility the initial vehicle was 2 hops away and as it gets closer 


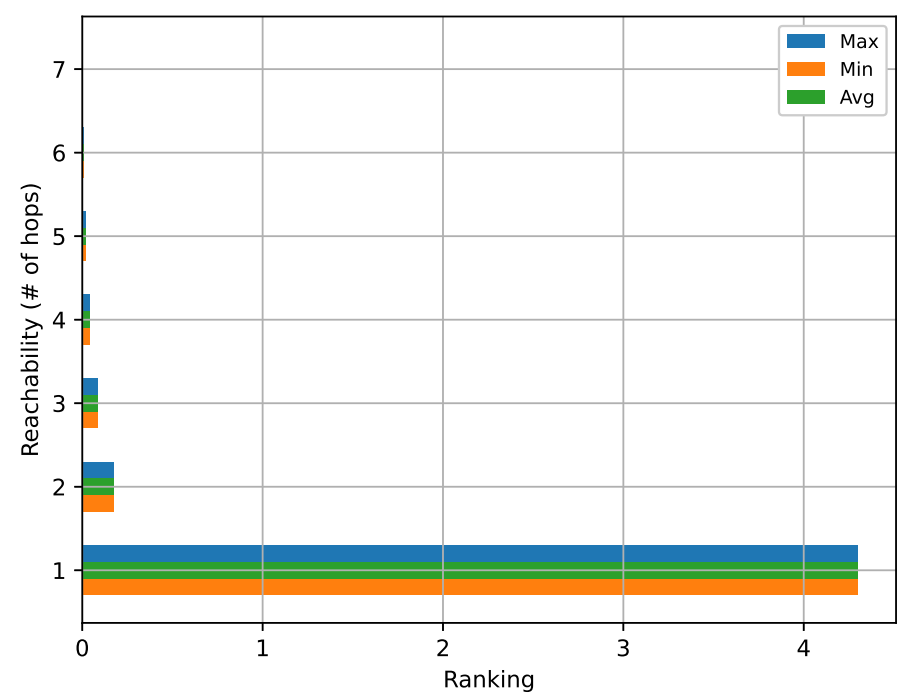

Figure 6.5: Vehicles head for destination. $(\gamma=1)$

to the RSU, changing its network distance to only 1 hop away. Consequently, the values of this vehicle increases. Vehicles are always moving around; as they either get close or far away from the RSU, the model assign the value and based on the ranking. Thus, vehicles might be selected as the "successful" candidate as the connectivity between the vehicles and RSU increases or the vehicles could also move futher away from the RSU or even other vehicles and be not reachable at all, hence, vehicles will be "unsuccessful" on the connectivity chart.

These results will stay constant if there is no change in topology of the vehicles. However, if there does exist a change in topology, MDP-model evaluates the nodes in different way as well. Figure 6.5 shows that difference compare to Figure $6.3 \mathrm{c}$. Just to simplify, we will focus mostly on density of 1000 vehicles within the same map. Figure 6.5, each vehicles only able to reach RSU one time, then it leaves the simulation. If that happens, MDP-model evaluate these nodes only once and that leads to lower ranking value. Notice the max, min, and average of one hop are all the same, even for two hop and so forth, because vehicles gets evaluated per hop and if the MDP-model notice that vehicles are within that hop, it does assign the similar value. Since, there is no revisit from these nodes back to RSU and the nodes transit there stay to be not connected, hence they leave the simulation. Figure 6.6 also shows similarity with Figure 6.5. Even when hops are increased to the 8, rewards of the nodes keep on deceasing as MDP-model evaluates these based on the number of hops. These topologies are still not completely random. We will see this in next 


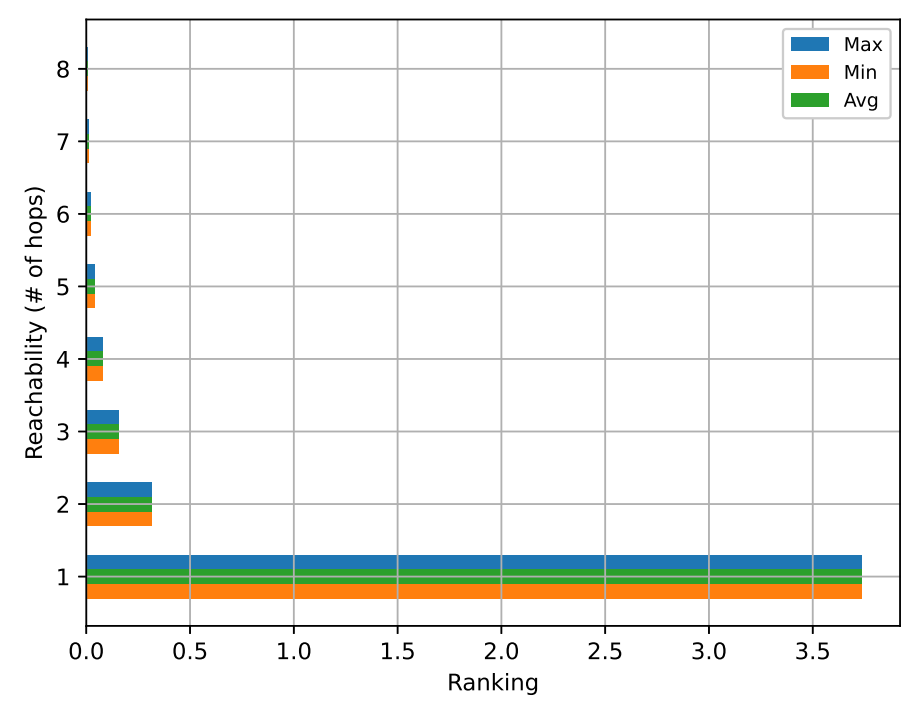

Figure 6.6: Vehicles head for destination. $(\gamma=1)$

sets of experiments as when topology does become completely random, MDP-model does evaluate them very different even on the same set of parameters.

The results in Figure 6.7, 6.8, and 6.9 are based on same parameters, such as, same discount factor, same map, and same density. The main difference here is the mobility of the vehicle is randomly generated. This causes MDP-model to behave in the drastically different in each of the simulations. Figure 6.7, display that nodes were in the constant connection, the mobility on the nodes was not as high as in other environment. Even up to eight hops, they nodes maintained there connected stay with RSU. The reason that even up to eight hops, the ranking of the nodes does not get effected as much, because the past probability taken into account for this simulation was $50 \%$. Hence, resulting in more stable connection even up to eight hops.

Mostly, as hops increases the MDP-model should evaluate nodes based on the hops as chances of disconnection gets higher, that can be noticed from figure 6.8 and 6.9. These nodes seems to only connected or be in the range of RSU once at most in figure 6.8. Since, the topology is randomly generated, it is possible that nodes are only evaluated once by the MDP-model. Figure 6.9 does not seems to have this case. There seems to be nodes that have higher connections time with only one hop. As the hops increase the connection becomes shaky, deceasing ranking of nodes greater than one hop away. These expirements is to prove MDP-model is definitely influenced by the mobility of the vehicles, but our goal is to find the best resource within this 


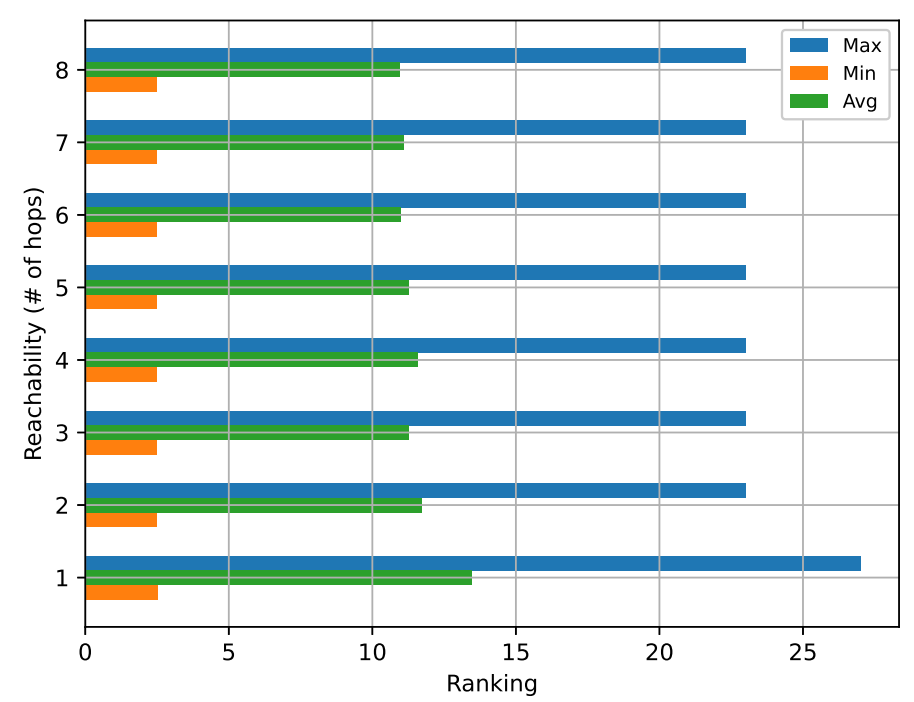

Figure 6.7: MDP-model ranking involving random mobility of vehicles. $(\gamma=1)$

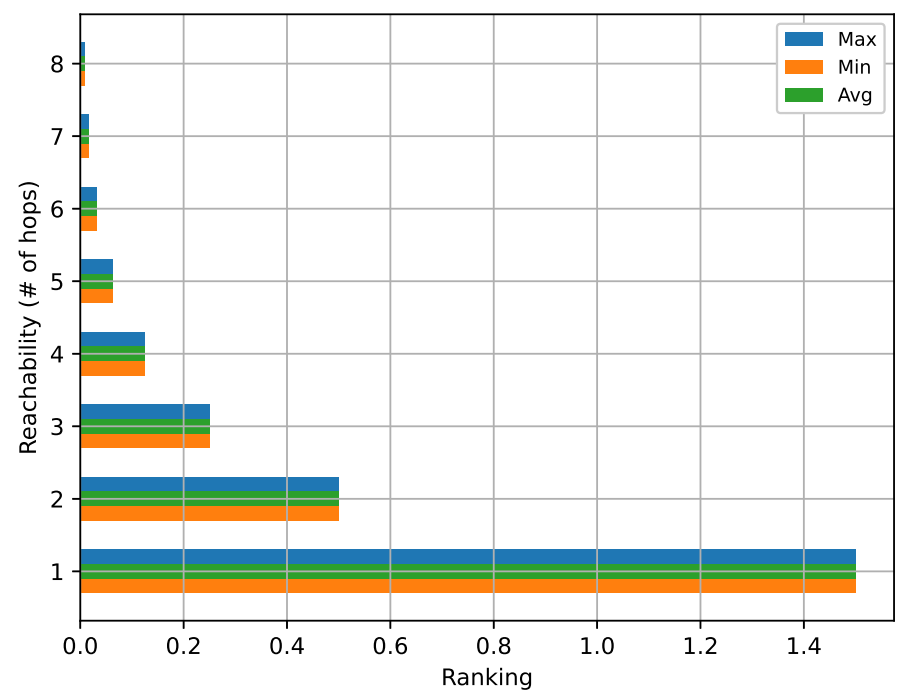

Figure 6.8: MDP-model ranking involving random mobility of vehicles. $(\gamma=1)$

network and these results does show MDP-model working.

The positioning of the RSU also plays an important role for stability of the connection and ranking of the nodes. As shown in the figure $6.2 \mathrm{a}$, these were area of the map, that had high density of vehicles. Figure 6.10 shows two graphs shows position of RSU in two different density achieving almost similar results. MDP-model might not give best of the results just because the mobility of the vehicles is not that great 


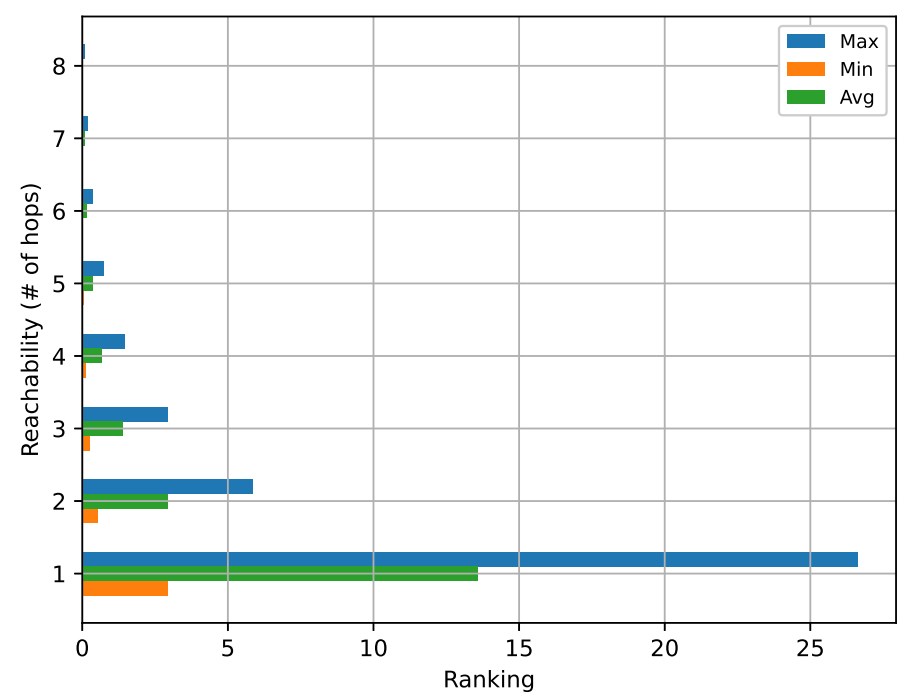

Figure 6.9: MDP-model ranking involving random mobility of vehicles. $(\gamma=1)$

in where this RSU has been placed. But, these ranking do exhibit similar traits as the figure 6.8 , because the position on the map is where the vehicles only seems to move in on direction and do not return, resulting in lowers the value of ranking. Just for the compeletion, figure $6.10 \mathrm{~b}$, shows if the density of the vehicles around the RSU is lowest, which results in lower ranking and lower number of hops. That is why positiong of the RSU and density of the vehicles do matter with the ranking of the vehicles. Higher density provides higher ranking values and higher number of connections in comparsion to the lower density exhibits lower ranking values and lower connections to the vehicles. This will become more adamant through the availability model experiments.

Additional experiments were conducted when LTE tower was developed into the simulation. Any vehicles that were close the LTE tower or within the range of LTE tower, selected that as a communication to the RSU. MDP-model should distinguish that the which vehicles has LTE state. LTE tower has more range and communication speed between the nodes quite faster in comparison to the RSU. When vehicle have LTE state, these vehicles do receives higher reward compare to the other states, because arriving at LTE state results in stable connection with the RSU. Figure 6.11 show that third hops has better average than second hop and even the first hop. Because, the location of the LTE tower is in high density area and vehicles from even the third hop away can reach the tower before the reaching RSU. Additionally, this graph highlights the essences of the LTE tower compares to RSU. The first hop are 


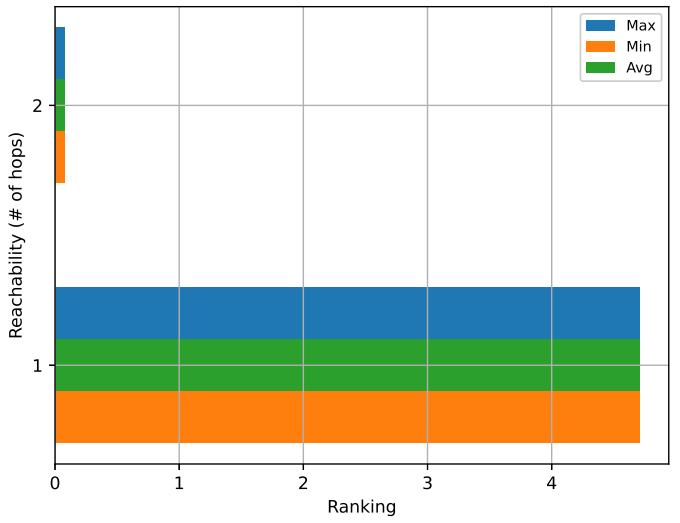

(a) $\gamma=1$

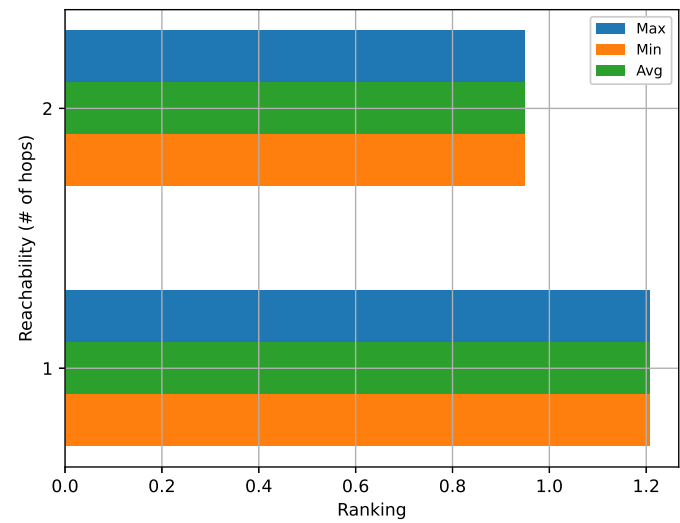

(b) $\gamma=1$

Figure 6.10: Ranking of vehicles in high density vs. lower density area

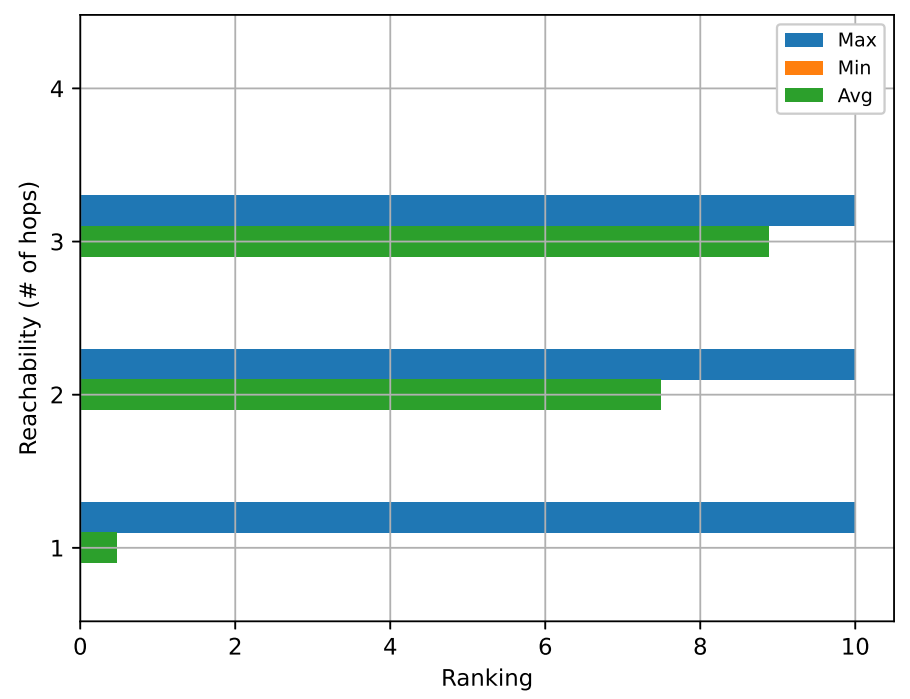

Figure 6.11: Node ranking with scenario including LTE Tower $(\gamma=0.1)$.

just the nodes close to the RSU, that do not have LTE tower in there communication range and the average on the second hop it just little shy of the third hop, because there are vehicles that reaching RSU, which are two hops away decreasing the average of the second hop. Fourth hop also does exist, but the values are just low enough for not to be on the scale. All of them includes min values as well.

The various discount factor does produces different results. In figure 6.12, the averages of each hops have changes in comparison to the pervious figure 6.11. The discount factor effects on the first hop increased the average and the minimum ranking, it is almost the same as the second hop average. The second hop has the highest 


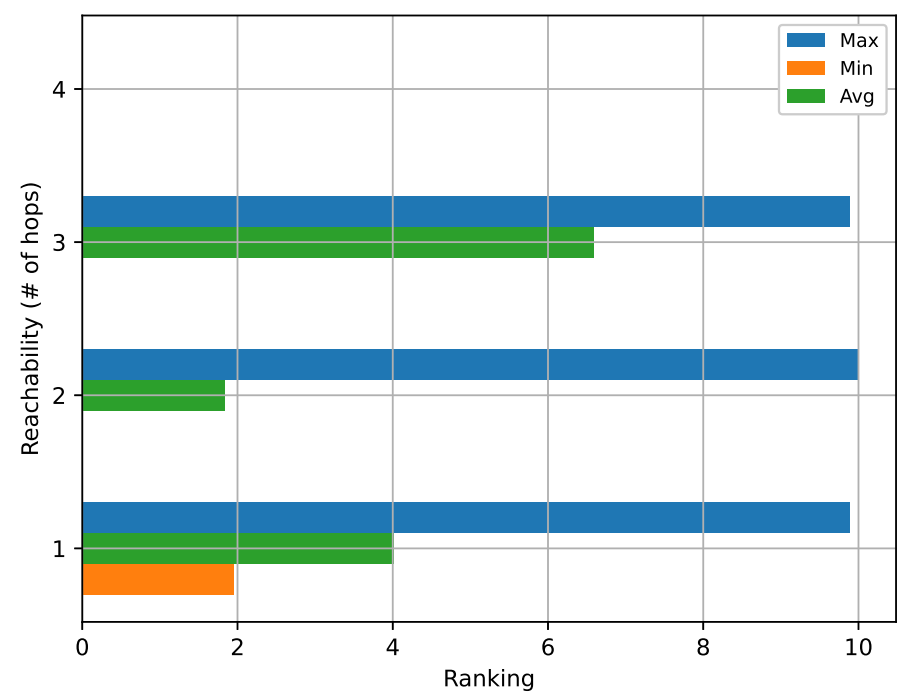

Figure 6.12: Node ranking with scenario including LTE Tower $(\gamma=0.5)$.

value among the hops, because the discount factor effects more on first and third hop. But, the average is still higher on the third hop, because of the higher density of vehicles in the LTE tower range. In comparison to figure 6.11, the average of the first hop has increased because of the higher discount factor resulting in a more communication from the nodes with state of V2R (vehicle-to-rsu). Some of the vehicles might also from transition from V2V (vehicle-to-vehicle) to V2R or V2L (vechicle-to-LTE).

These results show us the differences and similarities when comparing the discount factor and density. Mostly, the discount factor does not cause much of an effect when compared to the density, which shows a lot more relevance and impact on the model. This difference is more likely caused due to the assigned reward values in the model. Probably, a different range or scale of rewards might bring the discount factor with a stronger impact on the estimates. In our implementation scenario, if the state is not improving, the giving reward is negative and as the state begins to change the maximum reward (5) can be assigned. Hence, the discount factor does not seem to present a relevant effect. On the other hand, the impact of the density can be clearly seen in the graph from 500 to 2000. As the number of vehicles increases so does the value as the number of vehicles could vary possibly close to the RSU. As more and more vehicles travel, their state could move from V2V to V2I and that results in a better estimated connectivity value.

The second metric is based on the Success Rate. This determines if the vehicles is "connected" to the RSU at the end of the simulation. This also provides insight 


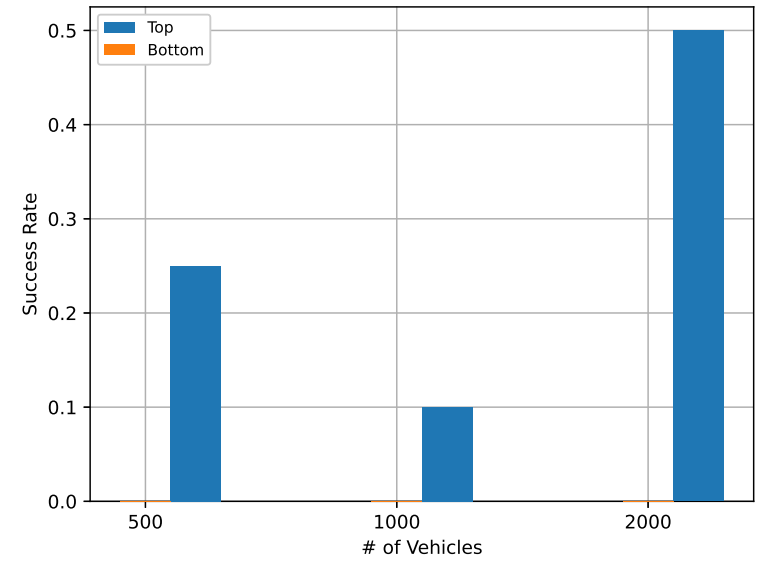

(a) $\gamma=0.1$

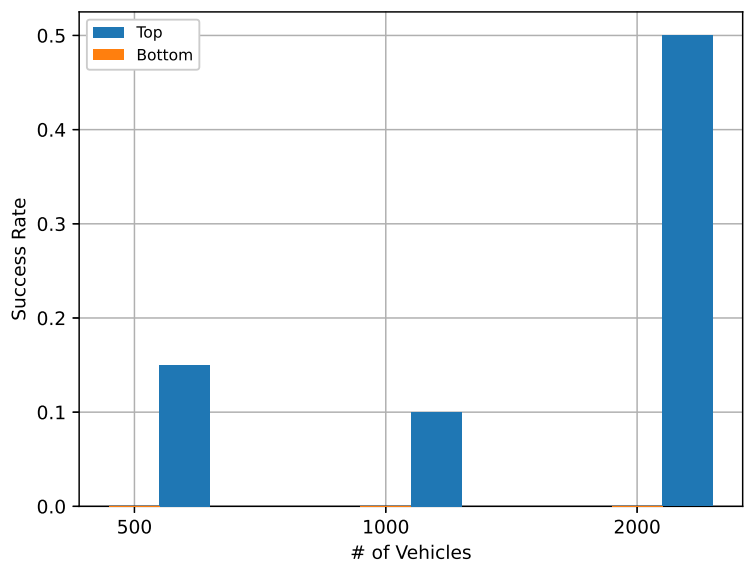

(b) $\gamma=0.5$

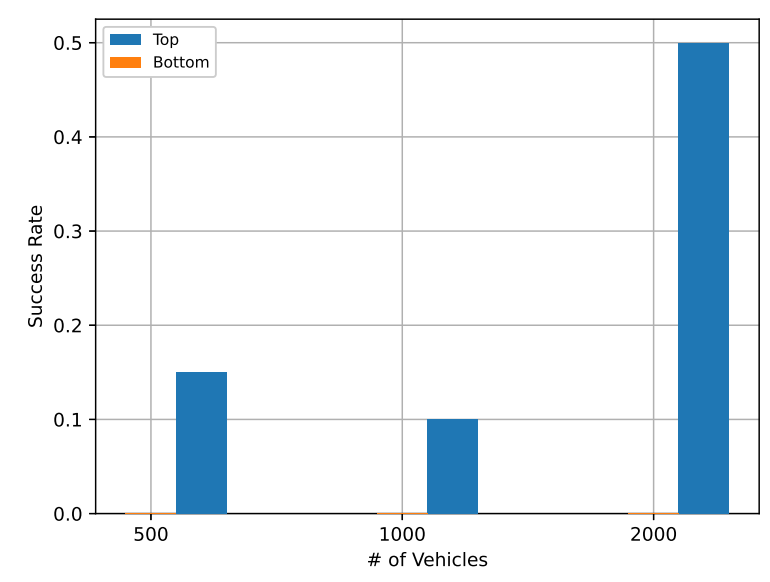

(c) $\gamma=1$

Figure 6.13: Ratio of successful connections.

of how long vehicles stay "connected" and if vehicle is within the range of RSU. The graphs represents Ratio of successful connections based on the destiny of the vehicles and how many vehicles contacted RSU at the end of the simulation. The ratio determines how many vehicles were in the map at the last $n$ number of second and was available for any type of connection with the RSU. Our aim is to find n "best" and "worst" vehicles from model and send them a message to see if these are still within the range of the RSU.

The Success Rate of the connections is summarized in Figures 6.13a, 6.13b, and 6.13c. It is expected that the top set shows a higher success ratio than the bottom set, because these graphs have same data as of graphs in figure 6.2 graphs. These graphs corresponds with the ranking of the nodes, graphs in 6.13 shows that mostly top ranking nodes communication is stable comparing to the bottom set, where there is 


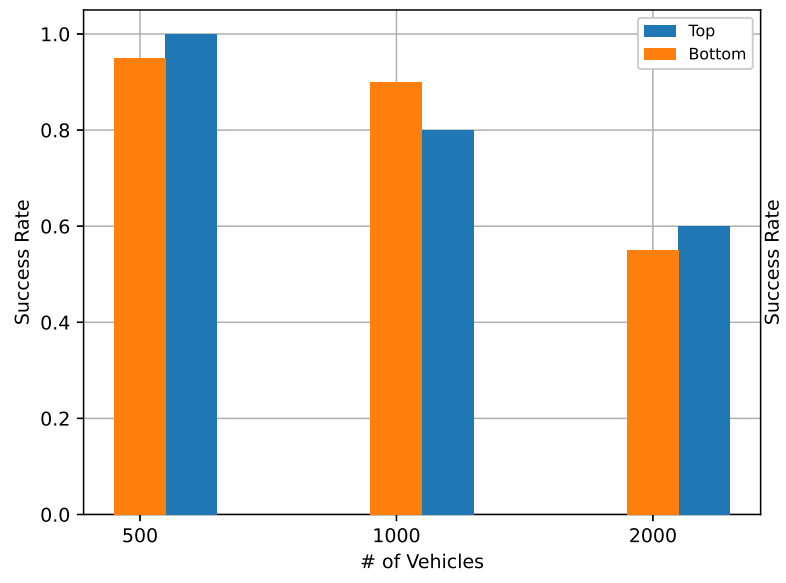

(a) $\gamma=0.1$

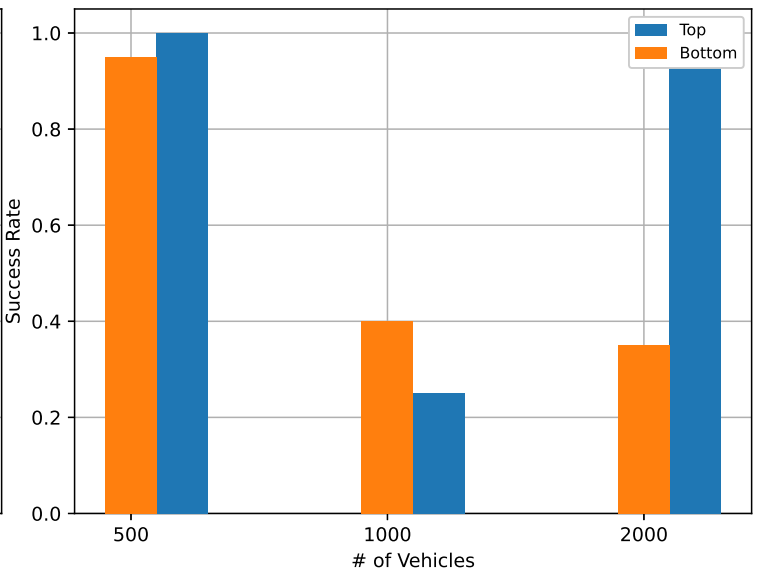

(b) $\gamma=0.5$

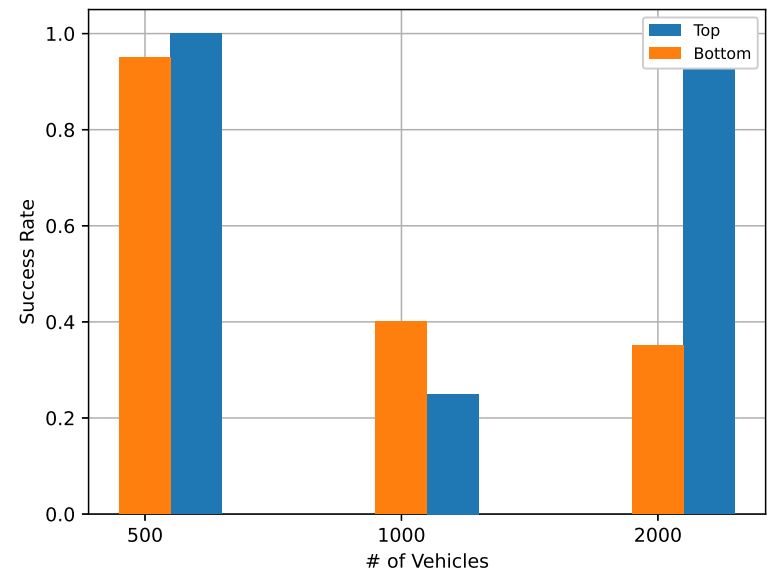

(c) $\gamma=1$

Figure 6.14: Ratio of successful connections show with different position of RSU.

no communication from the bottom set at all. Now, there has been case the bottom set ratios is better then the top ranking nodes. The reason behind a much lower success rate of the top group is high mobility. By the time contact messages are sent, vehicles have already left reach completely. Vehicles do not linger around the RSU, and they tend to leave since being in high mobility. Vehicles either leave the simulated area (map), the range of the RSU, or range of other vehicles. On the other hand, vehicles that were ranked low but moving towards the RSU were within its range when contacted.

Figure 6.14, shows the graphs of improved results when the RSU was moved to populated area. The movement of the vehicles are more repeatable in comparison from figure 6.13. Because, graphs in figure 6.13 only shows the top ranking vehicles having higher success ratio and density of the vehicles increase of have higher success 
ratio as well. In figure 6.14 the top set of vehicles have higher success ratio with discount factor of 0.5 and 1 in comparison to the 0.1 discount factor. The anomaly here is figure $6.14 \mathrm{a}$, higher density does not seem of the have higher success ratio, but success ratio is constant for 500 vehicles across all the graphs. Because, these 500 vehicles stay within the range of the RSU, that is also the reason why even the bottom set of vehicles have higher success ratio for this density.

After going through all these experiments and understanding the results, that lead us to believe MDP-model performances is based on the mobility and connectivity of the vehicles. So far we were researching the ranking of the vehicles and success ratio. These ranking were sort of forming a pattern everytime vehicles would connect to the RSU. These patterns can be determine through the use of another model: Availability. MDP-model focused towards the ranking of the vehicles and Availability-model will focus towards connectivity ratio of the vehicles to the RSU.

\subsection{Performance Analysis of Availability-Model}

This section reports on parameters, performance metrics and results attained from the Availability-model. Majortiy of the parameters for the simulation are listed in the pervious section, however availability-model entails additional parameters for computation of the ratio. Hence, new performance metrics have been included to support the pervious metrics and the results from MDP-model.

\subsubsection{Parameters}

To establish and constrict our experimental scenarios, additional parameters are incorporated and defined in combinations of parameter settings. Table 6.2 summarizes all the ranges of parameters used in our simulations.

In simulations, the scenarios and topology are sustained identical to the MDPmodel. Supplementary parameters comprise of LTE tower and LTE state, which is extends the mediums for communication and allows vehicles to be connected over V2I, V2V or V2X. The availability parameters, uptime denoted as $C_{\text {freq }}$ : frequency

of connections and downtime denote as $D_{\text {freq }}$ : frequency of disconnection, supports in computation of availability ratio for vehicles. Cycles is an additional parameter necessary for examining the MDP-model and availability-model. Alternatively, to MDP-model estimate the vehicles as soon as message is received, we collect states of the vehicles up to 3 cycles and then evaluate all the vehicles during that cycle. 
We performed experiments at cycle value of 3, because of simulation time being 1000 seconds, and periodic beacon happening every $40 \mathrm{sec}, 3$ cycles presented the best MDP-model results. Increasing the number of cycles permits MDP-model to run only few times, which was not enough for model to learn the patterns. This method enable us to develop some history for the MDP-model ranking. Remainder of the parameters, speed, RSU density, communication range, and discount factor are all maintained from the MDP-model parameters. The density of the vehicles has been set to 500, this authorize for the immediate inspection if the availability ratio is efficient for validating the MDP-model rankings of the vehicles.

For this particular simulation scenarios, a single RSU and LTE tower is deployed for the whole urban region. Consequently, during the simulation vehicles are necessarily out of range of the RSU, resorting to V2V or V2X communication or even being disconnected. For the simulations, the RSU is place at the bottom left quadrant of the region depicted in and LTE is replace right above the RSU in Figure 6.1.

Table 6.2: Simulation Parameter Settings

\begin{tabular}{rr}
\hline Parameter & Value Range \\
\hline Urban area & $1000 \times 1000 \mathrm{~m}^{2}$ \\
Vehicle density & 500 \\
Vehicle Speed & $5-15 \mathrm{~m} / \mathrm{s}$ \\
RSU density & 1 \\
LTE density & 1 \\
PHY model & IEEE $802.11 \mathrm{p}$ \\
Vehicle comm. range & $400 \mathrm{~m}$ \\
RSU comm. range & $400 \mathrm{~m}$ \\
Transmission power & $30 \mathrm{~mW}$ \\
$\gamma$ & $0.1,0.5,1$ \\
Transmission power LTE & $35 \mathrm{~mW}$ \\
Cycles & $3-5$ periodic beacon messages \\
$C_{\text {freq }}$ & $1-500$ frequency of connections \\
$D_{\text {freq }}$ & $1-500$ frequency of disconnections \\
\hline
\end{tabular}

Besides the different communication ranges for LTE tower and RSU adopted in our simulations, the transmission of vehicles, RSU, and LTE tower are susceptible to propagation, attenuation, and collision issues that might occur, according to the network simulator. Such issues are expected and needed so that the proposed model can properly represent dynamic conditions while estimating connectivity statuses of vehicles.

Since the availability model is supposed to adapt to changes with MDP-model 
results, the frequency of connections and frequency of disconnections will be recorded per cycle as the transition of vehicles are noted periodically. At the current implementation, RSU is the entity responsible for maintaining these frequencies for each vehicle. By the end of the simulation, all the frequency of connections and disconnections have been counted and that allows the RSU to calculate the availability ratio of each vehicle and rank them appropriately.

\subsubsection{Performance Metric}

There are three metrics employed do evaluate our availability model, Availability $R a$ tio, Ranking, and Success Ratio. The main performance metric known as, Availability Ratio estimates availability of the vehicles graded by MDP-model. The second metric, Ranking approximates the probability of connectivity status of the nodes through the MDP-model. The third metric, Success Ratio validates the success ratio of the vehicles through selecting top ranked vehicles vs. worst rank vehicles by reaching out in the last stages of the simulation. Lastly, the last two metrics advocate the results acquired from the availability ratio.

The main performance metric highlight in this section is designated as, Availability Ratio. This metric quantify $C_{\text {freq }}$ and $D_{\text {freq }}$ parameters to develop availability ratio. We rank these vehicles based on the availability ratio over the number of hops, similar to the Ranking metric adopted from the MDP-model analysis. If the vehicles have high availability ratio, that leads to higher connectivity to the RSU and higher ranking from the MDP-model. Vice-versa, if the vehicle has lower availability ratio, that conclude towards to lower connectivity to the RSU and lower ranking from the MDP-model. The cycles plays important role in understanding the availability ratio. Depending on the number of cycles, it changes the availability ratio of the vehicles. The rest of the metric presented in this section are to support the availability ratio anatomy.

This Ranking performance metric adopted from the MDP-model analysis to support the Availability Ratio metric. Ranking metric is maintained in this section, because this metric is effecitive in understanding the availability ratio. The vehicles with the constant "connectivity" have higher availability ratio, regardless of the "connectivity" state of the vehicles. The MDP-model acknowledges for four individual states: "Disconnected", "V2V", "V2I", and "V2X". The rewards for electing "V2X" communication results in higher rank, because that means the vehicles are connected to the RSU through LTE tower and the "connectivity" has strong connection, which 
ensure elevated availability ratio.

The Ranking metric is used to determine which vehicle can stay in constant contact, even if number of hops are increased. If the vehicle stays connected to the RSU any amount of data can be transferred to the vehicle and can be retrieved as well. The model picks the best candidate for the task. Thus Ranking metric proves to be more convenient. We can also compare the best top candidates with the lower worst candidates. Selecting the most "successful" candidates to distribute the data resulted in the faster delivery times of the request. The situation could also change, maybe the lower candidates becomes top as vehicles get closer to RSU, which is also why we picked the ranking system as our performance metric.

The Success Ratio of the most "successful" connections per number of vehicles is another metric observed. After being ranked, two sets of vehicles are contacted. The $\mathrm{n}$ vehicles with the highest ranks compose the top set while the $\mathrm{n}$ vehicles with the lowest ranks form the bottom set. We observe if the connections with the RSU persist or vehicles are reachable through $\mathrm{V} 2 \mathrm{~V}$ after they have reported their connectivity statuses through the sent messages. This metric helps establish an understanding of connectivity ratio in a high mobility environment. This will support the results obtained from the availability ratio, as contacted the most "successful" vehicles should have high availability ratio.

\subsubsection{Results}

This section is structured in explaining the results obtained from the MDP-model and supported by the availability-model. Initially, we start with initial MDP-model results, then we comprehend how availability-model supports the ranking of vehicles from MDP-model. The graphs are based on the $\mathrm{n}$ number of hops. Later in this analysis, we analysis more in depth of how each hop is effected and how per hop there is decreased in the value by the MDP-model.

The examination of figure 6.16a, points out how the min ranking for first three hops deviates in comparison to the rest of the hops. The reasoning for such a event, that RSU also receives communication from vehicles nearby or within the range of RSU, that decreased the min value of the vehicles ranked. There is also vehicles that leaves the simulation completely. The way the RSU is placed, there exist an exit in the lower quadrant of the map, that vehicles can take and leave the simulation. Even in this control environment it is not guaranteed, that some vehicles might not leave the simulation. Since, any communication that is not via V2L received a lower reward 


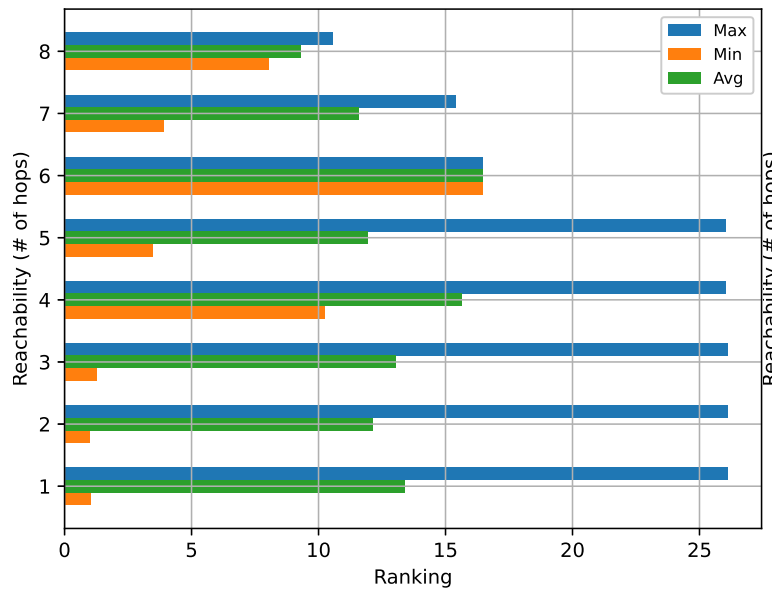

(a) $\gamma=0.1$

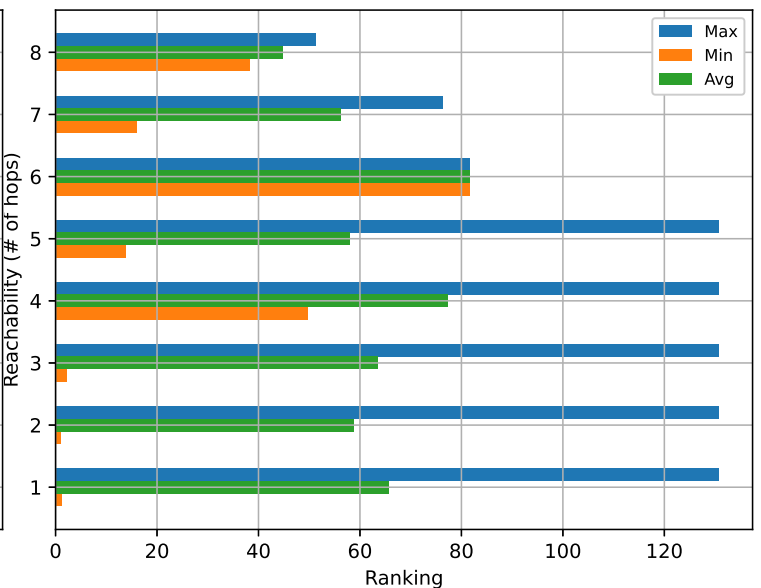

(b) $\gamma=0.5$

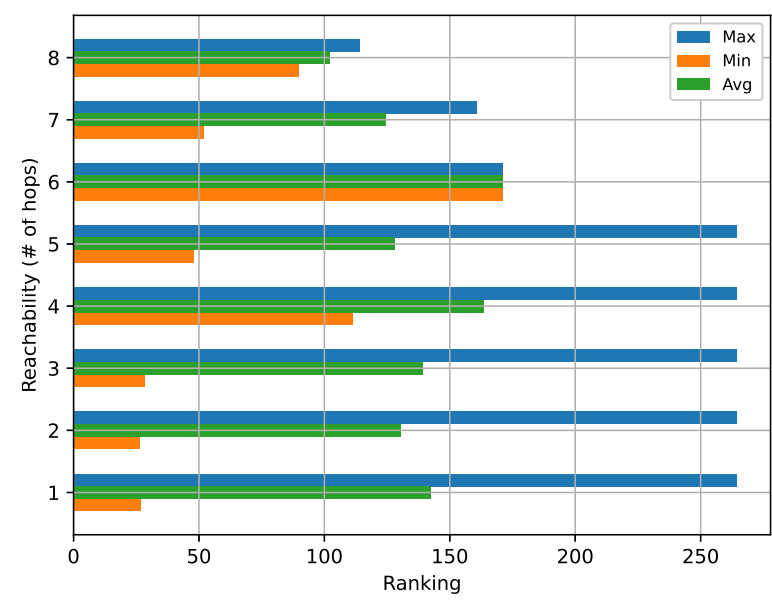

(c) $\gamma=1$

Figure 6.15: Ranking from MDP-model.

and vehicles further away from the RSU predominately communicate via $\mathrm{V} 2 \mathrm{~V}$ and V2L, hence, that caused the average to be better beyond the third hop.

There is another concern that is present in these graphs. The sixth hop always shows a consistence values for min, max, and average. Because, there is only one vehicle at the sixth hop and it does maintain the consist connection. This will also be verified with the availability ratio.

In Figures 6.15a, 6.15b, and 6.15c shows the ranking of the vehicles that are evaluated by the MDP-model. This experiments also included the LTE tower, which increased the covered area for the MDP-model to measure vehicles connectivity status. As shown by these graphs, the highest number of hops in the given scenario is reached up to eight hops. The discount factor matters in terms of ranking values of the vehicles. The higher discount factor results in more connections as the probability 


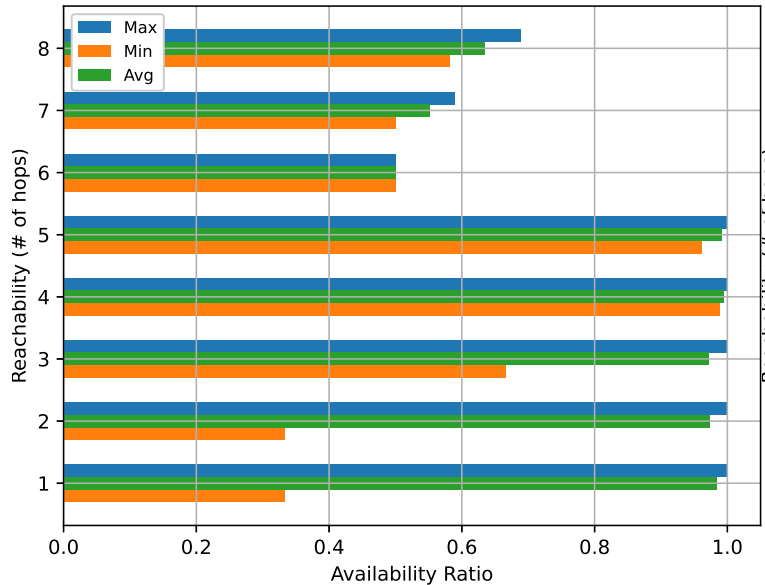

(a) $\gamma=0.1$

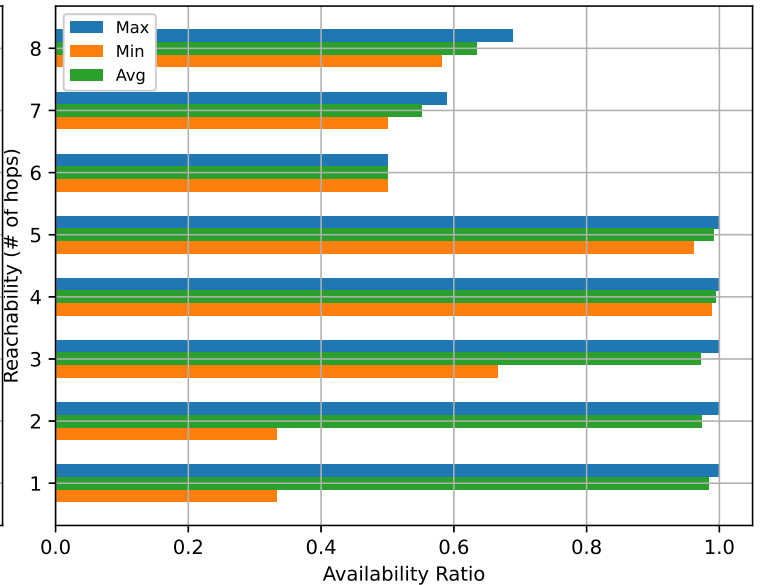

(b) $\gamma=0.5$

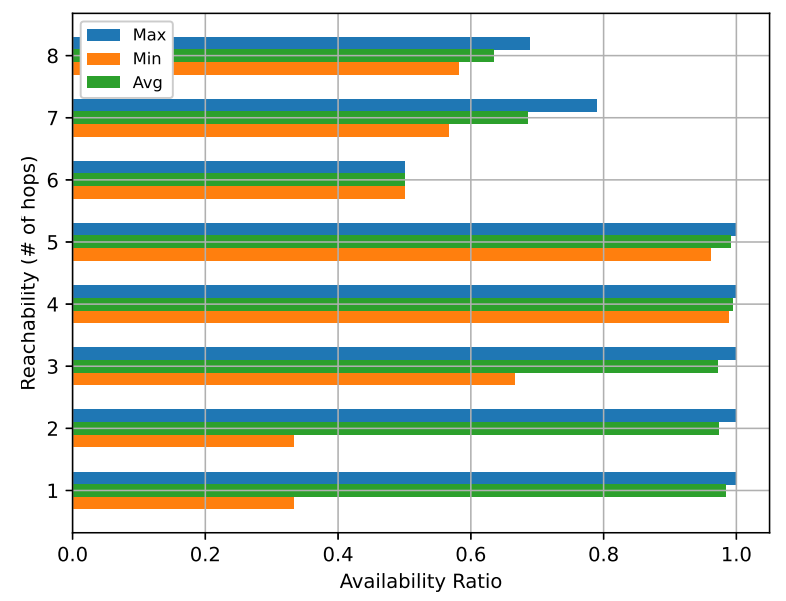

(c) $\gamma=1$

Figure 6.16: Availability Ranking.

of connections is much more stronger. Hence, connections become more stable up to five hops. After the fifth hop there is steep drop of the connection. Now, that could be blamed on the LTE tower. For example, lets say the LTE tower is about three or two hops away, once through V2V communication vehicles can hop to V2L and then V2R, that makes it five hops. Similar concept can be applied to anything less than fifth hops and the connection maintaining is recognized by the MDP-model.

The inspection of figure 6.16a supports the predication from the MDP-model. The ratio of availability follows the similar pattern as shown in the respective figure 6.15a. There is bit of difference with the sixth to eight hops. There are higher $D_{\text {freq }}$ (frequency of disconnections) that are happened within those hops. For the sixth hop, there was only one vehicle and the availability ratio is about $50 \%$, that happens if only $C_{\text {freq }}$ (frequency of connections) and $D_{\text {freq }}$ parameters are equal to 


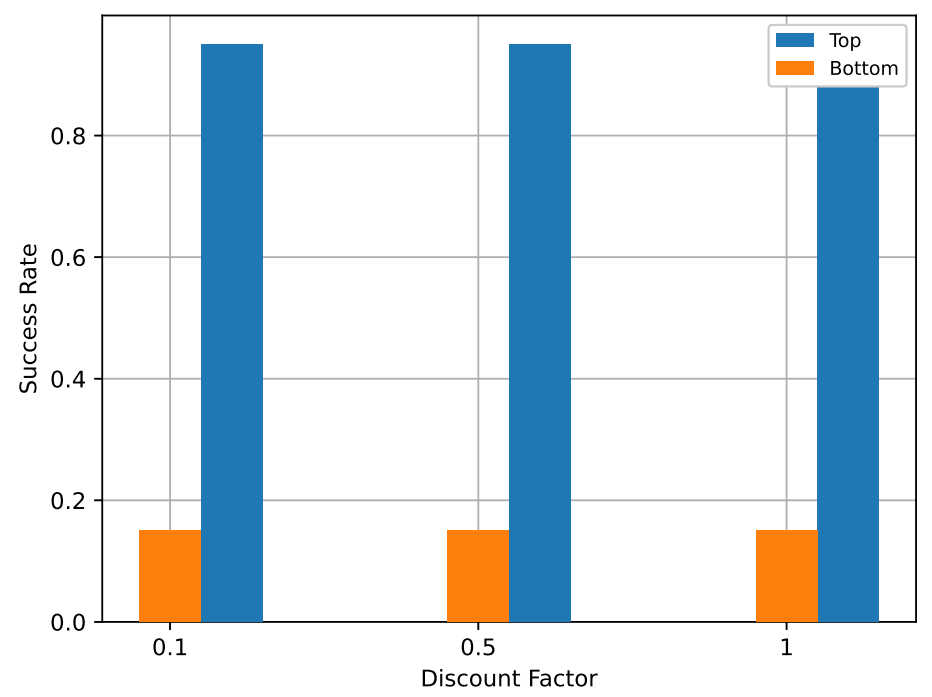

Figure 6.17: Success Ratio.

each other and that is also supported by the ranking from the MDP-model. The sixth hop ranking is almost half the highest ranking assigned to vehicles. The eight hops seem to have higher availability ratio than the seventh hop, because there are only three vehicles for the eight hop and $C_{\text {freq }}$ was higher then the $D_{\text {freq }}$ parameters. Because, the pattern showed it moved from the away from the fifth hop to the eight hop. Since, the $C_{f r e q}$ was higher in the fifth hop, that effects the availability ratio. This is known patterns among all the graphs.

The rest of the hops follows the predicated patterns from the availability ratio. In figures $6.16 \mathrm{~b}$ shows a predication similar to the figure $6.15 \mathrm{~b}$. Noticed how the min value for each ranking is lower and that is reflected in the availability ratio graphs as well. The max value for each hop is also repeated in the availability ratio graphs. The differences happened in figure 6.16c, the seventh hop has spiked in the availability ratio graph, but its not reflected in the same with MDP-model ranking in figure $6.15 \mathrm{c}$. It is because of the mobility of the vehicles from away from the LTE tower or the RSU increasing the $D_{\text {freq }}$ parameters.

The density of vehicles in figure 6.18 was 500 . The $\mathrm{x}$-axis is labeled with discount factor to reflect the graphs in figures 6.15 and 6.16. The success ratio supports the predication made by the availability ratio. For each discount factor, almost most of the top vehicles successfully replied back to the RSU by the end of the simulation. That states that the connections with these vehicles was stable. Figure 6.19 exhibits availability ratio against the discount factor. This graph reflects upon the top ranking 


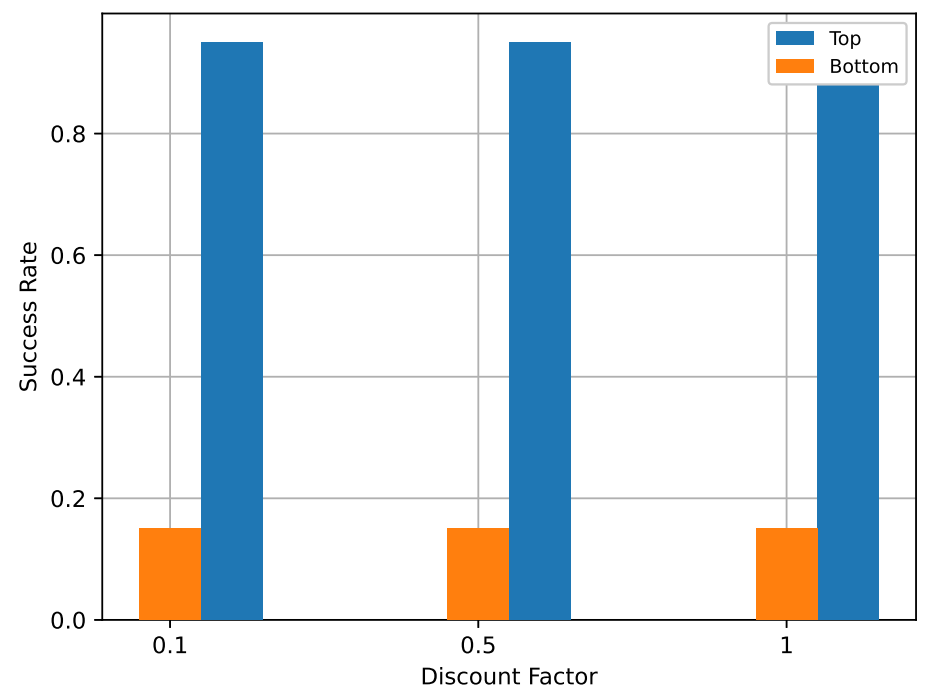

Figure 6.18: Success Ratio.

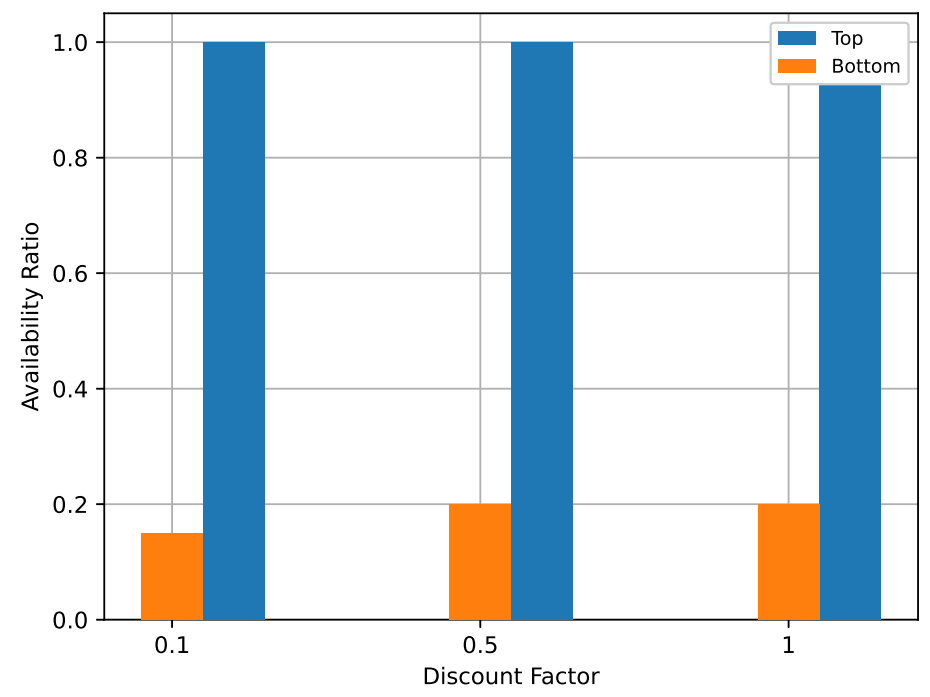

Figure 6.19: Availability Ratio.

nodes and checks the availability opposing the bottom ranking nodes. Reasonably, the top ranking nodes shows availability ratio of $100 \%$ proving availability ranking.

Figure 6.20 graphs demonstrates no repeating patterns among the vehicles within the network. This operates under the worst case scenarios. The rationale behind the MDP-model ranking these vehicles quite lower compare to graphs in figure 6.15, because of high mobility of the vehicles and little to no connectivity established 


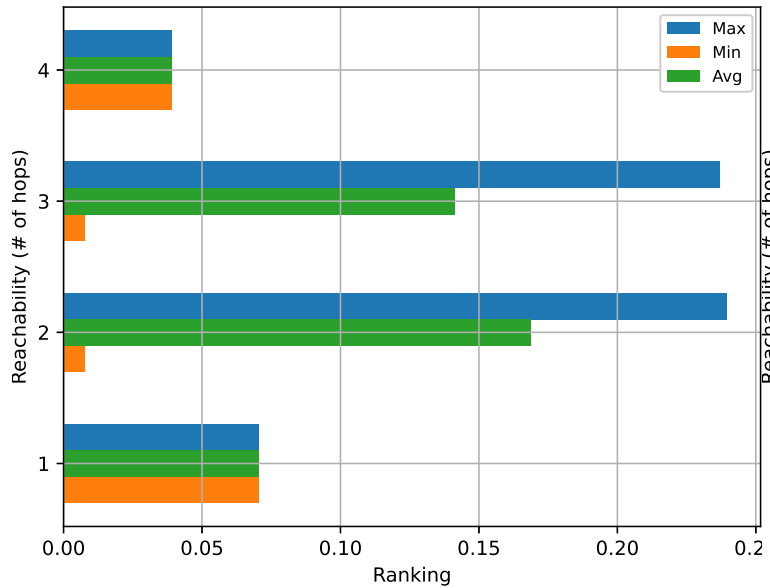

(a) $\gamma=0.1$

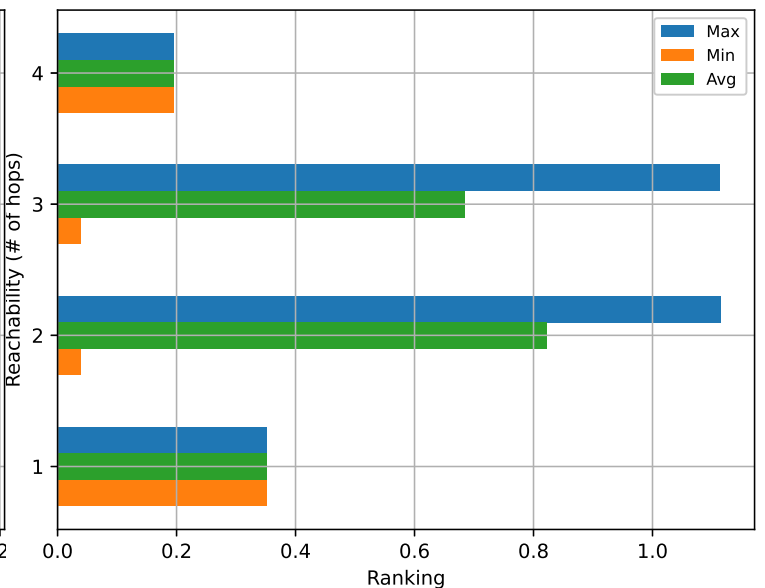

(b) $\gamma=0.5$

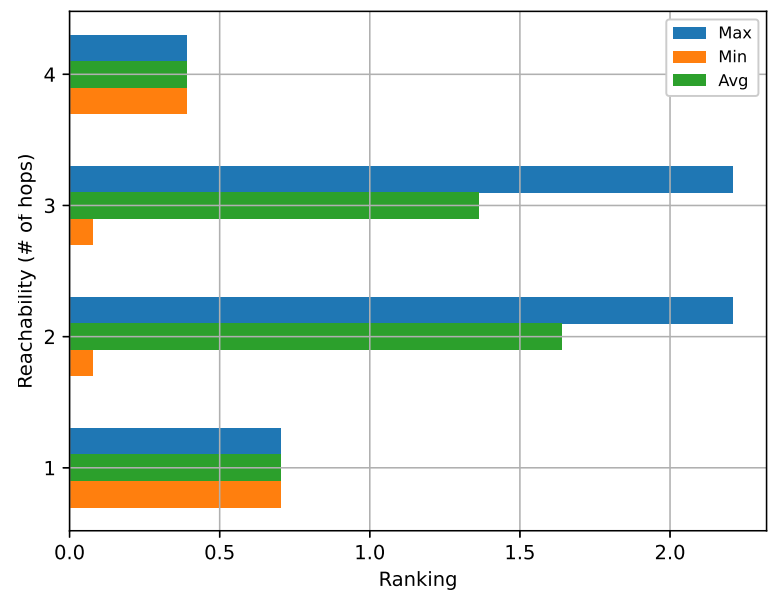

(c) $\gamma=1$

Figure 6.20: Ranking from MDP-model Worse Case.

with the vehicles. These nodes had awfully volatile movements and there was no guaranteed that they stayed in the simulation. The discount factor did effect the value of the ranking scale slightly, however, the increase in discount factor had a narrow impact on the connectivity with nodes. Since, the connectivity status of vehicles had no recurrent patterns MDP-model results are jusified. Vehicles travelled in straight or followed a random movement patterns, hence, the MDP-model ranking emerged as poorest in this scenario.

The outcomes delivered in figure 6.20 graphs was contemplative in graphs of figure 6.21. These graphs presents the number of hops over availability ratio. The first hop was $10 \%$ of availability ranking, that is represented by only single vehicle. The second and third hop had higher availability ratio, because of the LTE tower range covered further area in comparison to the RSU. The reason behind higher availability 


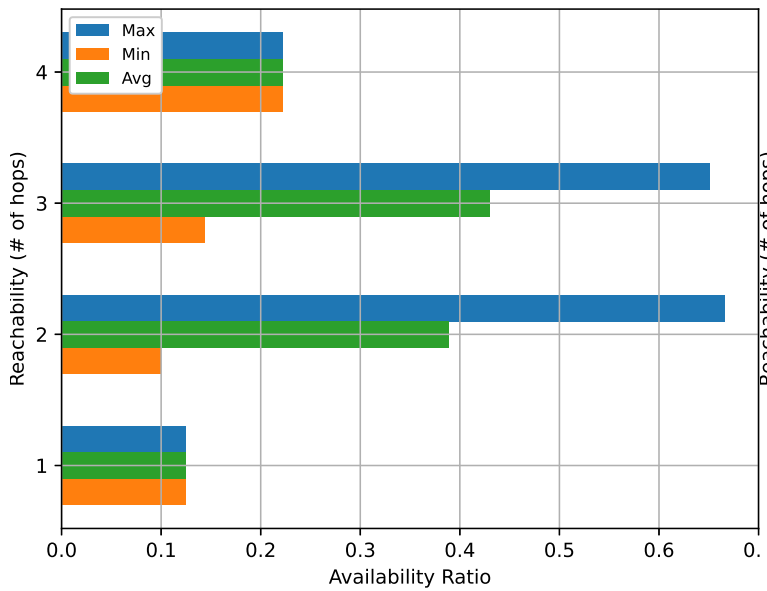

(a) $\gamma=0.1$

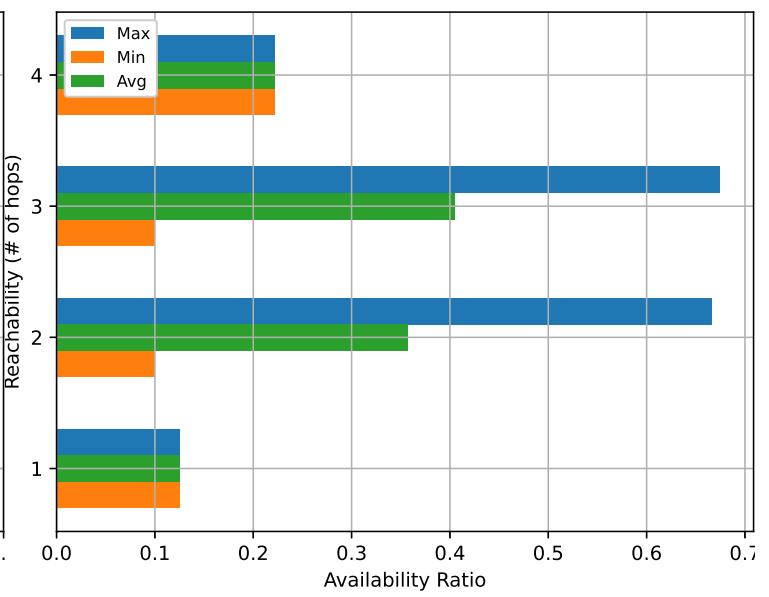

(b) $\gamma=0.5$

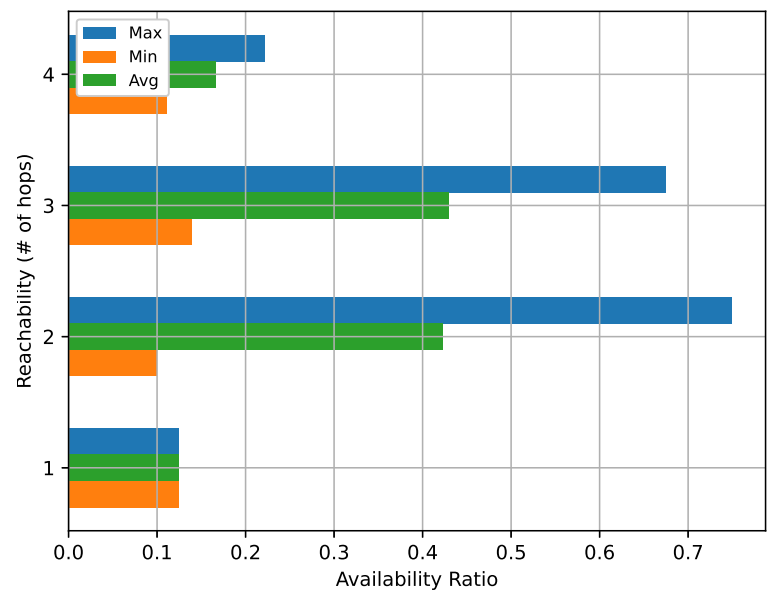

(c) $\gamma=1$

Figure 6.21: Availability Ranking Worse Case.

ratio, because of only few number of vehicles connected to the LTE and as these vehicles moves away, but not further away that communication completely faded away. The availability ranking for second and third hop evaluated to be $40 \%$, which is reflected from the MDP-model ranking. Similar to the first hop, fourth hop is represented by only single vehicle as well, a little higher connectivity in comparison to the first hop. Since, availability ranking exhibits the similar pattern as in the graphs of figure 6.20.

Further examining of the success ratio in figure 6.22 shows that low number of vehicles were available to communicate by the end of the simulation. The $30 \%$ of top ranking vehicles managed to connect to the RSU in last stage of the simulation for success ratio. There are $10 \%$ of bottom ranking vehicles that succeed in connectivity in the last interval. This statistics validates our availability ranking and MDP-model 


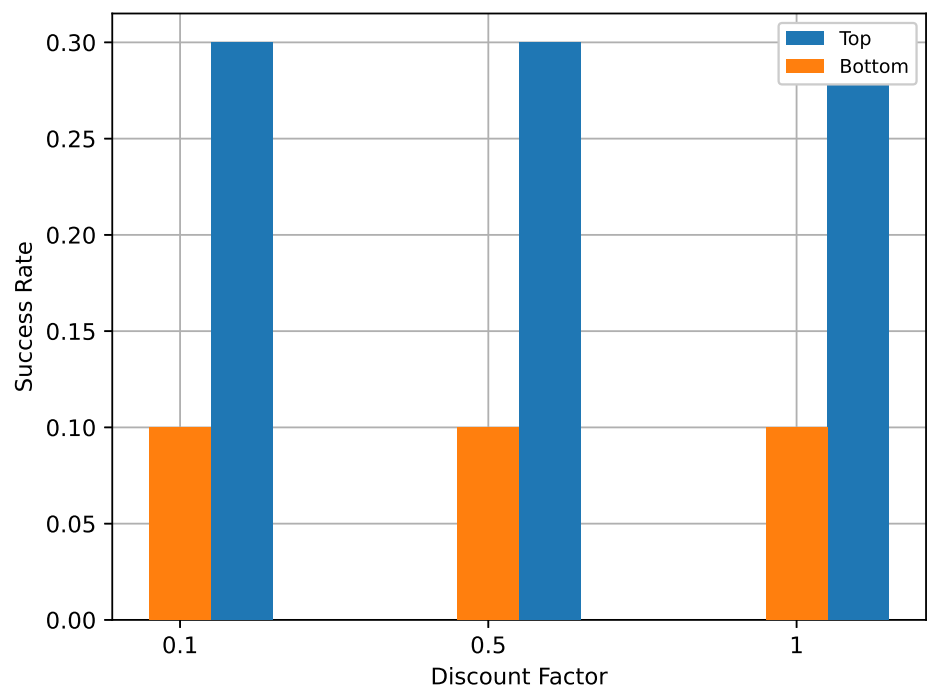

Figure 6.22: Success Ratio.

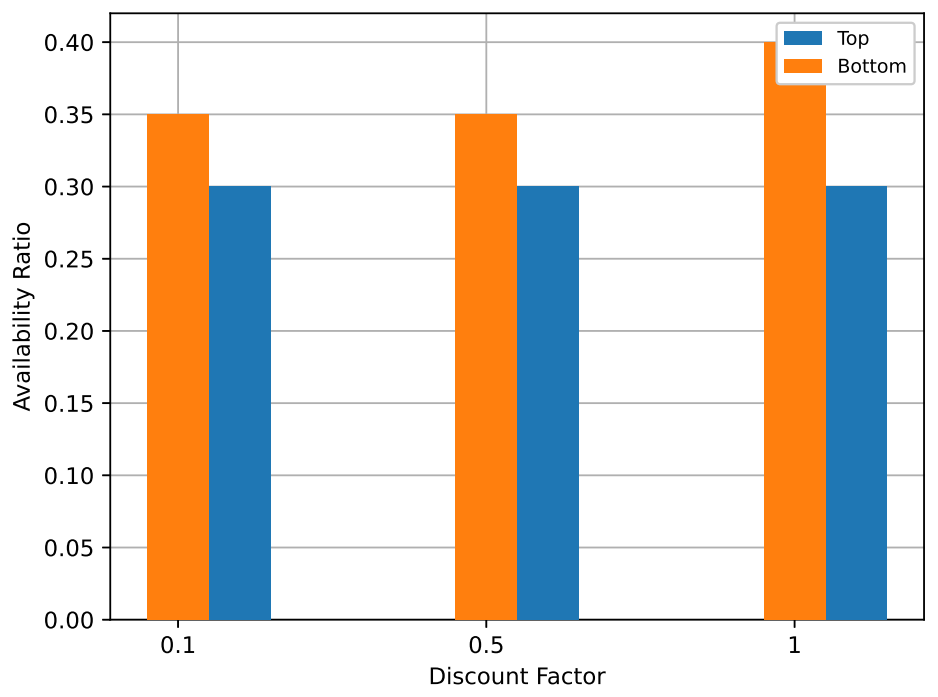

Figure 6.23: Availability Ratio.

ranking. The scale was low, however there are some vehicles, that had the ability to connect and somewhat poorly maintain the connection to the LTE tower or the RSU. Additional metric of availability ratio validates the success ratio in figure 6.23. 


\subsection{Summary}

In essence, MDP-model could correctly represents the connectivity status of the vehicles presuming there is repeatedly amongst the vehicles to sustain the constant connection. MDP-model delivers more accurate estimates of the ranking if vehicles maintains connection often. However, the model is ineffective if vehicles do not revisit connection status as there exist no pattern for MDP-model to precisely determine the rank of the vehicles based on connectivity. Therefore, Availability-model has been proposed to identify the suitability of the MDP-model by measure the vehicles availability. This proposed model computes the availability ratio of the vehicles to establish the ranks from MDP-model. Higher availability ratio generates higher ranking from the MDP-model as the connectivity status from the vehicles is justified. 


\section{Chapter 7}

\section{Conclusion}

In this paper, we have proposed a new models for estimating and representing the connectivity level in vehicular networks. The connectivity model optimistically identifies the "best" vehicle candidate in a scenario where the access to vehicles permeates the delivery of services and resources in a Vehicular Cloud fashion. We have evaluated the proposed models through simulations, which showed that the ranking properly represents the connectivity conditions of vehicles.

\subsection{Summary of Contributions}

In this work, the proposed MDP-based model evaluated the connectivity level of the vehicles in the network. MDP-model ranked these resources based on the connectivity status of the nodes in the vehicular networks. The vehicles communicated to RSU or LTE tower through harnessing the orthodox communications of V2V, V2I, or V2X. The initial state of these vehicles were set to "not connected", when vehicles in mobility had a method to dispatch a message, the states transitioned into V2V, V2R, or V2L. These states supported the MDP-model in determining the communication method applied and rewarded accordingly. The ranking shown in graphs of figure 6.1, 6.2, and 6.3 analyzed through Ranking and Success Ratio metrics established the most 'successful' resources within the vehicular network. To satisfy the results obtained from the MDP-model, we proposed availability-model to verfiy the rankings of the vehicles.

Availability-model estimates the uptime and downtime of the services measured in the distributed systems. Additional model was proposed to compute the availability of the resources ranked by the MDP-model. In this work, the availability-model estimated the probability of connections and disconnections for each vehicles in the 
vehicular network. The two scenario experimented in this work described as high availability against the low availability. The high availability scenario depicted in figure 6.15, in which high ratios of vehicles lingers within the map, resulted in increased number of connections. This is confirmed in figure 6.16, presented with analyzed graph of Availability-ratio metric, therefore highly ranked from MDP-model, that justified the suitability MDP-model. The second scenario illustrated in figure 6.19 , consisted of lower ratios of vehicles that remains within the map, ensured the lower number of connections. This is confirmed in figure 6.20 through analyzing the Availability-ratio, hence lower ranked from the MDP-model and rightfully the analysis confirmation of non suitability of the MDP-model.

There do exist limitations of this work. The foremost limitation is caused by the flooding in this network. Increasing the number of vehicles increases the time requires for the simulation to be conclude, because the flooding the network increasing the communication time among the vehicles. Flooding also causes more collision within the networks, that increases the backoff time of the resources to communicate amongst each other or with RSU or with LTE tower. The second limitation its the processing power required for compute the simulation results. As of now, the map is about $1 \mathrm{~km}$ $\mathrm{x} 1 \mathrm{~km}$, but if the size of the map increasing, that will require more computing power to sustain the number of vehicles on that map. My equipment does not allow for the that level of computation as of now. Lastly, there is quite a stress on RSU to compute both models and handling on the messages from all forms of communications. This impacts the time of the simulation through slowing the process down for evaluating all the resources.

\subsection{Future Research Directions}

The collision within the network could never be completely eliminated, however reducing the number of collision could provide for improved results. Applying more suitable flooding protocol can reduce the collision and the backoff time. The communication can improve within the vehicular network and influencing finer tune results. Diminishing the stress from the RSU and extracting the models to compute on different module lessens the time simulation take to complete. In return more experiments can be performed, therefore improving the results. Lastly, acquiring upgraded equipment for experiments can greatly effects the simulation times and permits for exceeding the map or density of vehicles limits to apply this work on grander scale.

There are few research that can be spun of from this work. A Reinforcement- 
learning model could be applied to analyze structure paths of connected resources and in predicating there movements for better 'connectivity status'. Same reinforcementlearning model could be utilize to discover shortest path to the resources for improving the connectivity and reducing the number of hops.

Developing more suitable flooding protocols for the network benefits commuication speeds among the vehicles, RSU and LTE tower. That increasing the probability of identifying 'successful' resources accurately. Expanding the number of RSU or LTE tower for covering large scale maps. Finally, incorporation of $5 \mathrm{G}$ communication will grant a jump in the communication speed, as well as reduces the flooding and the amount of data transfer to the resources. 


\section{Bibliography}

[1] Saif Al-Sultan, Moath M. Al-Doori, Ali H. Al-Bayatti, and Hussien Zedan. A comprehensive survey on vehicular ad hoc network. Journal of Network and Computer Applications, 37:380 - 392, 2014.

[2] Ashwin Ashok, Peter Steenkiste, and Fan Bai. Vehicular cloud computing through dynamic computation offloading. Elsevier Computer Communications, 120:125 - 137, 2018.

[3] Y. Bi, H. Zhou, W. Xu, X. S. Shen, and H. Zhao. An efficient pmipv6-based handoff scheme for urban vehicular networks. IEEE Transactions on Intelligent Transportation Systems, 17(12):3613-3628, 2016.

[4] A. Boukerche, R. W. L. Coutinho, and X. Yu. Lisic: A link stability-based protocol for vehicular information-centric networks. In Proceeding of the IEEE International Conference on Mobile Ad Hoc and Sensor Systems, pages 233-240, 2017.

[5] Azzedine Boukerche and Robson E. De Grande. Vehicular cloud computing: Architectures, applications, and mobility. Elsevier Computer Networks, 135:171 - 189, 2018.

[6] S. Choo, I. Jang, J. Koo, J. Kim, and S. Pack. Reliable vehicle selection algorithm with dynamic mobility of vehicle in vehicular cloud system. In IEEE Asia-Pacific Network Operations and Management Symposium, pages 319-321, 2017.

[7] Rodolfo W. L. Coutinho, Azzedine Boukerche, and Xiangshen Yu. Informationcentric strategies for content delivery in intelligent vehicular networks. In Proceeding of the ACM Symposium on Design and Analysis of Intelligent Vehicular Networks and Applications, pages 21-26, 2018.

[8] Praneet Dhingra and Prem Chand Jain. Cost-effective vertical handoff strategies in heterogeneous vehicular networks. In Proceedings of the Springer International 
Conference on Advanced Computational and Communication Paradigms, pages 369-377, 2018.

[9] J. A. F. F. Dias, J. J. P. C. Rodrigues, F. Xia, and C. X. Mavromoustakis. A cooperative watchdog system to detect misbehavior nodes in vehicular delaytolerant networks. IEEE Transactions on Industrial Electronics, 62(12):79297937, 2015.

[10] Ryan Florin and Stephan Olariu. Toward approximating job completion time in vehicular clouds. IEEE Transactions on Intelligent Transportation Systems, PP:1-10, 112018.

[11] K. K. Ghanshala, S. Sharma, S. Mohan, L. Nautiyal, P. Mishra, and R. C. Joshi. Self-organizing sustainable spectrum management methodology in cognitive radio vehicular adhoc network (cravenet) environment: A reinforcement learning approach. In 2018 First International Conference on Secure Cyber Computing and Communication (ICSCCC), pages 168-172, 2018.

[12] Ying He, F. Richard Yu, Zhexiong Wei, and Victor Leung. Trust management for secure cognitive radio vehicular ad hoc networks. Ad Hoc Networks, 86:154 $-165,2019$.

[13] Issam Jabri, Tesnim Mekki, Abderrazak Rachedi, and Maher Ben Jemaa. Vehicular fog gateways selection on the internet of vehicles: A fuzzy logic with ant colony optimization based approach. Ad Hoc Networks, page 101879, 2019.

[14] S. Kaur, K. Kaur, and D. Singh. A framework for hosting web services in cloud computing environment with high availability. In 2012 IEEE International Conference on Engineering Education: Innovative Practices and Future Trends (AICERA), pages 1-6, 2012.

[15] S. Khan Tayyaba, H. A. Khattak, A. Almogren, M. A. Shah, I. Ud Din, I. Alkhalifa, and M. Guizani. 5g vehicular network resource management for improving radio access through machine learning. IEEE Access, 8:6792-6800, 2020.

[16] Yunxin (Jeff) Li. An overview of the dsrc/wave technology. In Xi Zhang and Daji Qiao, editors, Quality, Reliability, Security and Robustness in Heterogeneous Networks, pages 544-558, Berlin, Heidelberg, 2012. Springer Berlin Heidelberg. 
[17] C. Lin, D. Deng, and C. Yao. Resource allocation in vehicular cloud computing systems with heterogeneous vehicles and roadside units. IEEE Internet of Things Journal, 5(5):3692-3700, 2018.

[18] Pablo Alvarez Lopez, Michael Behrisch, Laura Bieker-Walz, Jakob Erdmann, Yun-Pang Flötteröd, Robert Hilbrich, Leonhard Lücken, Johannes Rummel, Peter Wagner, and Evamarie Wießner. Microscopic traffic simulation using sumo. In The 21st IEEE International Conference on Intelligent Transportation Systems. IEEE, 2018.

[19] S. Mehar, S. M. Senouci, A. Kies, and M. M. Zoulikha. An optimized roadside units (rsu) placement for delay-sensitive applications in vehicular networks. In Proceeding of the Annual IEEE Consumer Communications and Networking Conference, pages 121-127, 2015.

[20] Mohamed Nidhal Mejri, Jalel Ben-Othman, and Mohamed Hamdi. Survey on vanet security challenges and possible cryptographic solutions. Vehicular Communications, 1(2):53 - 66, 2014.

[21] Rodolfo Ipolito Meneguette and Azzedine Boukerche. Servites: An efficient search and allocation resource protocol based on $\mathrm{V} 2 \mathrm{~V}$ communication for vehicular cloud. Elsevier Computer Networks, 123:104-118, 2017.

[22] Rodolfo Ipolito Meneguette, Azzedine Boukerche, and Robson Eduardo De Grande. SMART: an efficient resource search and management scheme for vehicular cloud-connected system. In Proceeding of IEEE GLOBECOM, pages 1-6, 2016.

[23] Rodolfo Ipolito Meneguette, Azzedine Boukerche, Adinovam H. M. Pimenta, and Messias Meneguette. A resource allocation scheme based on semi-markov decision process for dynamic vehicular clouds. In Proceeding of the IEEE International Conference on Communications, ICC, pages 1-6, 2017.

[24] Felipe Modesto and Azzedine Boukerche. A novel service-oriented architecture for information-centric vehicular networks. In Proceeding of the ACM International Conference on Modeling, Analysis and Simulation of Wireless and Mobile Systems, pages 136-139, 2016.

[25] A. M. Mustafa, O. M. Abubakr, O. Ahmadien, A. Ahmedin, and B. Mokhtar. Mobility prediction for efficient resources management in vehicular cloud com- 
puting. In Proceedings of the IEEE International Conference on Mobile Cloud Computing, Services, and Engineering, pages 53-59, 2017.

[26] Mina Nabi, Maria Toeroe, and Ferhat Khendek. Availability in the cloud: State of the art. Journal of Network and Computer Applications, 60:54-67, 2016.

[27] Ali Kazemi Niari, Reza Berangi, and Mahmood Fathy. Eccn: an extended ccn architecture to improve data access in vehicular content-centric network. The Journal of Supercomputing, 74(1):205-221, 2018.

[28] F. H. Rahman, A. Yura Muhammad Iqbal, S. H. S. Newaz, A. Thien Wan, and M. S. Ahsan. Street parked vehicles based vehicular fog computing: Tcp throughput evaluation and future research direction. In 2019 21st International Conference on Advanced Communication Technology (ICACT), pages 26-31, 2019.

[29] J. Ren, Y. Zhang, K. Zhang, and X. Shen. Exploiting mobile crowdsourcing for pervasive cloud services: challenges and solutions. IEEE Comm. Magazine, 53(3):98-105, 2015.

[30] Ryan Reynolds. Achieving 'five nines' in the cloud for justice and public safety. https://aws.amazon.com/blogs/publicsector/ achieving-five-nines-cloud-justice-public-safety/, 2020.

[31] Claude Sammut and Geoffrey I. Webb, editors. Bellman Equation, pages 97-97. Springer US, Boston, MA, 2010.

[32] Christoph Sommer, Reinhard German, and Falko Dressler. Bidirectionally Coupled Network and Road Traffic Simulation for Improved IVC Analysis. IEEE Transactions on Mobile Computing, 10(1):3-15, 12011.

[33] M. Sookhak, F. R. Yu, Y. He, H. Talebian, N. Sohrabi Safa, N. Zhao, M. K. Khan, and N. Kumar. Fog vehicular computing: Augmentation of fog computing using vehicular cloud computing. IEEE Vehicular Technology Magazine, 12(3):55-64, 2017.

[34] N. Tamani, B. Brik, N. Lagraa, and Y. Ghamri-Doudane. On link stability metric and fuzzy quantification for service selection in mobile vehicular cloud. IEEE Transactions on Intelligent Transportation Systems, pages 1-13, 2019.

[35] András Varga and Rudolf Hornig. An overview of the omnet++ simulation environment. In Proceedings of the 1st International Conference on Simulation 
Tools and Techniques for Communications, Networks and Systems 83 Workshops, Simutools '08, pages 60:1-60:10. ICST (Institute for Computer Sciences, SocialInformatics and Telecommunications Engineering), 2008.

[36] Y. Wang, J. Zheng, and N. Mitton. Delivery delay analysis for roadside unit deployment in vehicular ad hoc networks with intermittent connectivity. IEEE Transactions on Vehicular Technology, 65(10):8591-8602, 2016.

[37] Md Whaiduzzaman, Mehdi Sookhak, Abdullah Gani, and Rajkumar Buyya. A survey on vehicular cloud computing. Journal of Network and Computer Applications, 40:325-344, 2014.

[38] Changqiao Xu, Wei Quan, Athanasios V. Vasilakos, Hongke Zhang, and GabrielMiro Muntean. Information-centric cost-efficient optimization for multimedia content delivery in mobile vehicular networks. Elsevier Computer Communications, 99:93 - 106, 2017.

[39] F. Zhang, R. E. De Grande, and A. Boukerche. Macroscopic interval-split freeflow model for vehicular cloud computing. In Proceeding of the IEEE/ACM International Symposium on Distributed Simulation and Real Time Applications, pages 1-8, 2017. 